Pacific

Journal of

Mathematics

\title{
HANDLE ADDITIONS PRODUCING ESSENTIAL SURFACES
}

RUIFENG QIU AND SHICHENG WANG 


\title{
HANDLE ADDITIONS PRODUCING ESSENTIAL SURFACES
}

\author{
RUIFENG QIU AND SHICHENG WANG
}

\begin{abstract}
We construct a small, hyperbolic 3-manifold $M$ with the property that, for any integer $g \geq 2$, there are infinitely many separating slopes $r$ in $\partial M$ such that the 3-manifold $M(r)$ obtained by attaching a 2-handle to $M$ along $r$ contains an essential separating closed surface of genus $g$. The resulting manifolds $M(r)$ are still hyperbolic. This contrasts sharply with known finiteness results on Dehn filling and with the known finiteness result on handle addition for the cases $g=0,1$. Our 3-manifold $M$ is the complement of a hyperbolic, small knot in a handlebody of genus 3.
\end{abstract}

\section{Introduction}

All manifolds in this paper are orientable and all surfaces $F$ in 3-manifolds $M$ are embedded and proper, unless otherwise specified. A surface $F \subset M$ is proper if $F \cap \partial M=\partial F$.

Let $M$ be a compact 3-manifold. An incompressible, $\partial$-incompressible surface $F$ in $M$ is essential if it is not parallel to $\partial M$. A 3-manifold $M$ is simple if $M$ is irreducible, $\partial$-irreducible, anannular and atoroidal. In this paper, a compact 3manifold $M$ is said to be hyperbolic if $M$ with its toroidal boundary components removed admits a complete hyperbolic structure with totally geodesic boundary. By Thurston's theorem, a Haken 3-manifold is hyperbolic if and only if it is simple. A knot $K$ in $M$ is hyperbolic if $M_{K}$, the complement of $K$ in $M$, is hyperbolic. A 3-manifold $M$ is small if $M$ contains no essential closed surface. A knot $K$ in $M$ is small if $M_{K}$ is small.

A slope $r$ in $\partial M$ is an isotopy class of unoriented essential simple closed curves in $F$. We denote by $M(r)$ the manifold obtained by attaching a 2-handle to $M$ along a regular neighborhood of $r$ in $\partial M$ and then capping off the possible spherical component with a 3-ball. If $r$ lies in a toroidal component of $\partial M$, this operation is known as Dehn filling.

MSC2000: primary 57M25; secondary 57M50.

Keywords: Hyperbolic knot, small knot, handle additions.

Both authors are supported by the National Science Foundation of China. 
Essential surfaces are a basic tool in the study of 3-manifolds, and handle addition is a basic method to construct 3-manifolds. A central question connecting those two topics is the following:

Question 1. Let $M$ be a hyperbolic 3-manifold with nonempty boundary, containing no essential closed surface of genus $g$. How many slopes $r \subset \partial M$ are there such that $M(r)$ contains an essential closed surface of genus $g$ ? (The question is asked only for hyperbolic 3-manifolds to avoid possibly infinitely many slopes produced by Dehn twists along essential discs or annuli. The mapping class group of a hyperbolic 3-manifold is finite.)

The main result of this paper shows that there can be many such slopes:

Theorem 1. There is a small, hyperbolic knot $K$ in a handlebody $H$ of genus 3 such that, for any given integer $g \geq 2$, there are infinitely many separating slopes $r$ in $\partial H$ such that $H_{K}(r)$ contains an essential separating closed surface of genus $g$. Moreover the resulting manifolds $H_{K}(r)$ are still hyperbolic.

Remarks. Let $M$ be a hyperbolic 3-manifold with nonempty boundary.

(1) Suppose $\partial M$ is a torus. W. Thurston's pioneer result [1982] asserts that there are at most finitely many slopes on $\partial M$ such that $M(r)$ is not hyperbolic; hence the number of slopes in Question 1 is finite when $g=0$ or 1 . Sharp upper bounds for this number were given by Gordon and Luecke for $g=0$, and by Gordon for $g=1$; see the survey paper [Gordon 1997]. Hatcher [1982] proved that the number is finite for any $g$.

(2) Suppose $\partial M$ has genus at least 2. Scharlemann and Wu [1993] have shown that if $g=0$ or 1, there are only finitely many separating slopes $r$ such that $M(r)$ contains an essential closed surface of genus $g$. Recently Lackenby [2002] generalized Thurston's finiteness result to handlebody attaching, proving that, for a hyperbolic 3-manifold $M$, there is a finite set $C$ of exceptional curves on $\partial M$ such that attaching a handlebody to $M$ yields a hyperbolic-like manifold if none of those curves bounds a meridian disc of the handlebody.

(3) In [Qiu and Wang 2005] we proved Theorem 1 for $g$ even.

Theorem 1 and the finiteness results just cited give a global view about the answer of Question 1.

Outline of the proof of Theorem 1 and organization of the paper. In Section 2 we first construct a knot $K$ in the handlebody $H$ of genus 3 for Theorem 1, then we construct infinitely many surfaces $S_{g, l}$ of genus $g$ for each $g \geq 2$ such that (1) all those surfaces are disjoint from the given $K$, hence contained in $H_{K}$; and (2) for fixed $g$, all the $\partial S_{g, l}$ are connected and provide infinitely many slopes in $\partial H$ as $l$ varies. Those $\partial S_{g, l}$ will serve as the slopes $r$ in Theorem 1. We denote 


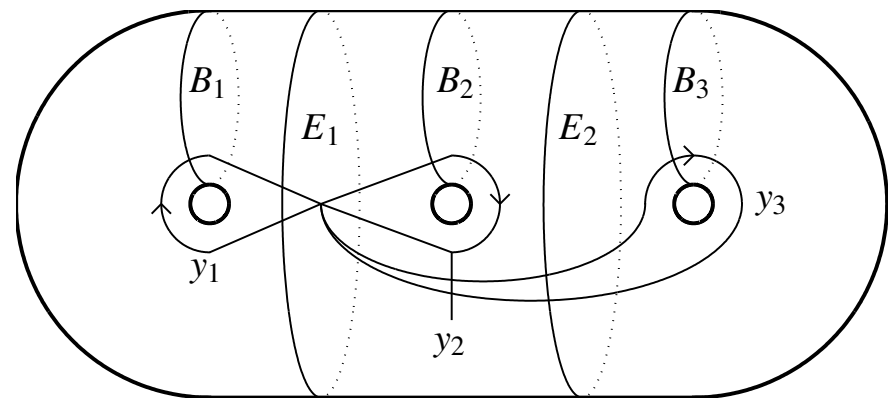

Figure 1

by $\hat{S}_{g, l} \subset H_{K}\left(\partial S_{g, l}\right)$ the closed surface of genus $g$ obtained by capping off the boundary of $S_{g, l}$ with a disk. We will prove in Section 3 that $\hat{S}_{g, l}$ is incompressible in $H_{K}\left(\partial S_{g, l}\right)$. In Sections 4 and 5 we prove that the knot $K$ is hyperbolic and small.

\section{Construction of the knot $K$ and the surfaces $S_{g, l}$ in $H$}

Let $H$ be a handlebody of genus 3. Suppose that $B_{1}, B_{2}$ and $B_{3}$ are basis disks of $H$, and $E_{1}, E_{2}$ are disks in $H$ that separate $H$ into three solid tori $J_{1}, J_{2}$ and $J_{3}$. See Figure 1.

Let $c$ be a closed curve in $\partial H$ as in Figure 2. The boundary of $E_{1} \cup E_{2}$ separates $c$ into 10 arcs $c_{1}, \ldots, c_{10}$, where $c_{1}, c_{3}, c_{9} \subset J_{1}$ meet $B_{1}$ in two, one, one points respectively; $c_{2}, c_{4}, c_{6}, c_{8}, c_{10} \subset J_{2}$ meet $B_{2}$ in one, one, two, zero, one points respectively; $c_{5}, c_{7} \subset J_{3}$ meet $B_{3}$ in one, three points respectively.

Let $u_{1}, \ldots, u_{2 g}, v_{1}, \ldots, v_{2 g}$ be $4 g$ points located on $\partial E_{1}$ in the cyclic order $u_{1}$, $u_{3}, \ldots, u_{2 i-1}, \ldots, u_{2 g-1}, u_{2 g}, u_{2 g-2}, \ldots, u_{4}, u_{2}, v_{1}, v_{3}, \ldots, v_{2 i-1}, \ldots v_{2 g-1}, v_{2 g}$, $v_{2 g-2}, \ldots, v_{4}, v_{2}$ as in Figure 3 . In view of the order of these points, $C$ can be

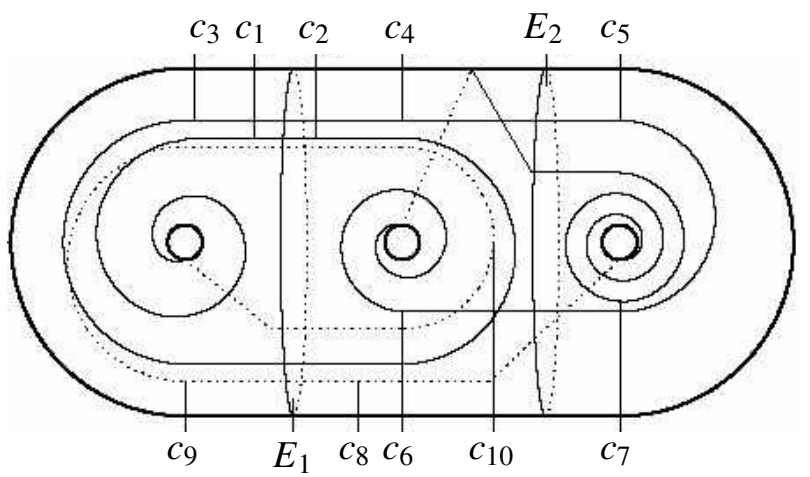

Figure 2 




Figure 3

isotoped so that $\partial c_{1}=\left\{u_{1}, v_{1}\right\}, \partial c_{2}=\left\{u_{1}, v_{2}\right\}, \partial c_{10}=\left\{v_{1}, u_{2}\right\}, \partial c_{3}=\left\{v_{2}, u_{3}\right\}$, $\partial c_{9}=\left\{u_{2}, v_{3}\right\}$. Now suppose $u_{2 i+1} v_{2 i}$ and $v_{2 i+1} u_{2 i}$, for $1 \leq i \leq g-1$, are arcs in $\partial J_{1}-\stackrel{\circ}{E}_{1}$ parallel to $c_{3}$ and $c_{9}$, and that $u_{2} v_{1}=c_{10}, v_{2} u_{1}=c_{2}$, and $u_{2 i} v_{2 i-1}, v_{2 i} u_{2 i-1}$, for $2 \leq i \leq g$, are parallel arcs in $\partial\left(J_{2} \cup J_{3}\right)-\stackrel{\circ}{E}_{1}$, each of which intersects $B_{2}$ in one point and $B_{3}$ in $l$ points (see Figure 3, where $l=2$ ). Finally define $\alpha_{1}=u_{1} v_{1}$, and let $\alpha_{k}$ be the union of $v_{k-1} u_{k}, \alpha_{k-1}$ and $u_{k-1} v_{k}$, for $k=2, \ldots, 2 g$. Then $\alpha_{1} \subset \alpha_{2} \subset \cdots \subset \alpha_{2 g}$ is an increasing sequence of arcs.

Let $\alpha \subset \partial H$ be an arc which meets $\partial S$ exactly at its two endpoints for a proper separating surface $S \subset H$. The surface resulting from tubing $S$ along $\alpha$ in $H$, denoted by $S(\alpha)$, is obtained by first attaching a 2-dimensional 1-handle $N(\alpha) \subset$ $\partial H$ to $S$, then making the surface $S \cup N(\alpha)$ to be proper, that is, pushing its interior into the interior of $H$. The image of $N(\alpha)$ after the pushing is still denoted by $N(\alpha)$. In fact, $S \cup N(\alpha)$ is a once punctured torus. Since $S$ is orientable and separating, $S(\alpha)$ is still separating and orientable.

Since $\alpha_{1}$ meets $E_{1}$ exactly in its two endpoints, we do tubing on $E_{1}$ along $\alpha_{1}$ to get a proper surface $E_{1}\left(\alpha_{1}\right)$. Now $\alpha_{2}$ meets $E_{1}\left(\alpha_{1}\right)$ exactly in its two endpoints. We do tubing on $E_{1}\left(\alpha_{1}\right)$ along $\alpha_{2}$ to get $E_{1}\left(\alpha_{1}, \alpha_{2}\right)=E_{1}\left(\alpha_{1}\right)\left(\alpha_{2}\right)$, where the tube $N\left(\alpha_{2}\right)$ is thinner and closer to $\partial H$ so that it goes over the tube $N\left(\alpha_{1}\right)$. Hence $E_{1}\left(\alpha_{1}, \alpha_{2}\right)$ is a properly embedded surface (indeed, a one-punctured torus). By the same argument, we do tubing along $\alpha_{3}, \ldots, \alpha_{2 g}$ to get a proper embedded surface $E_{1}\left(\alpha_{1}, \ldots, \alpha_{2 g}\right)$ in $H$, denoted by $S_{g, l}$. This surface is orientable and separating.

Since $S_{g, l}$ is obtained from the disc $E_{1}$ by attaching $2 g$ 1-handles to $E_{1}$ such that the ends of any two handles are alternating, $S_{g, l}$ is a once punctured orientable surface of genus $g$. We summarize the facts just discussed:

Lemma 2.1. $S_{g, l}$ is a once punctured surface of genus $g$ and is separating in $H$. 
Now let $K$ be a knot in $\stackrel{H}{H}$ obtained by first pushing $c_{6}$ into $\stackrel{H}{H}$ deeply and then pushing $C-c_{6}$ into $\stackrel{\circ}{H}$ so that it stays between $N\left(\alpha_{3}\right)$ and $N\left(\alpha_{4}\right)$. The following fact is clear:

Lemma 2.2. $K$ is disjoint from $S_{g, l}$ for all $g, l$.

\section{Proof of Theorem 1 assuming that $K$ is hyperbolic and small}

We denote by $\hat{S}_{g, l} \subset H_{K}\left(\partial S_{g, l}\right) \subset H\left(\partial S_{g, l}\right)$ the surface obtained by capping off the boundary of $S_{g, l}$ with a disk. Then $\hat{S}_{g, l}$ is a closed surface of genus $g$.

From the definition of $S_{g, l}$ for a given genus $g$, the boundary $\partial S_{g, l}$ provides infinitely many boundary slopes as $l$ varies from 1 to infinity. Then Theorem 1 follows from the next two propositions (apart from the last assertion, which follows directly from [Scharlemann and $\mathrm{Wu}$ 1993]).

Proposition 3.0. $K \subset H$ is a hyperbolic, small knot.

Proposition 3.1. $\hat{S}_{g, l}$ is incompressible in $H_{K}\left(\partial S_{g, l}\right)$.

We postpone the proof of the first of these results and prove the second here. Recall that a surface $F$ in a 3-manifold is compressible if either $F$ is a 2 -sphere that bounds a 3-ball, or there is an essential simple closed curve in $F$ that bounds a disk in $M$; otherwise, $F$ is incompressible. Hence Proposition 3.1 is a consequence of the following result:

Proposition 3.2. $\hat{S}_{g, l}$ is incompressible in $H\left(\partial S_{g, l}\right)$.

We choose the center of $E_{1}$ as the common base point for the fundamental groups of $H$ and of all surfaces $S_{g, l}$.

Now $\pi_{1}\left(S_{g, l}\right)$ is a free group of rank $2 n$ generated by $\left(x_{1}, \ldots, x_{2 n}\right)$, where $x_{i}$ is the generator given by the centerline of the tube $N\left(\alpha_{i}\right)$; and $\pi_{1}(H)$ is a free group of rank three generated by curves $y_{1}, y_{2}, y_{3}$ corresponding to $B_{1}, B_{2}, B_{3}$, as in Figure 1. Let $i: S_{g, l} \rightarrow H$ be the inclusion. One can read $i_{*}\left(x_{i}\right)$ directly as words in $y_{1}, y_{2}, y_{3}$ :

$$
\begin{aligned}
& i_{*}\left(x_{1}\right)=y_{1}^{2}, \\
& i_{*}\left(x_{2}\right)=y_{2} y_{1}^{2} y_{2}, \\
& i_{*}\left(x_{3}\right)=y_{1} y_{2} y_{1}^{2} y_{2} y_{1}, \\
& i_{*}\left(x_{4}\right)=y_{2} y_{3}^{l} y_{1} y_{2} y_{1}^{2} y_{2} y_{1} y_{2} y_{3}^{l},
\end{aligned}
$$

and in general, for $2 \leq i \leq g$,

$$
\begin{aligned}
i_{*}\left(x_{2 i-1}\right) & =y_{1}\left(y_{2} y_{3}^{l} y_{1}\right)^{i-2} y_{2} y_{1}^{2} y_{2}\left(y_{1} y_{2} y_{3}^{l}\right)^{i-2} y_{1}, \\
i_{*}\left(x_{2 i}\right) & =\left(y_{2} y_{3}^{l} y_{1}\right)^{i-1} y_{2} y_{1}^{2} y_{2}\left(y_{1} y_{2} y_{3}^{l}\right)^{i-1} .
\end{aligned}
$$

Lemma 3.3. $S_{g, l}$ is incompressible in $H$. 
The proof is the same as that in [Qiu 2000].

Now $S_{g, l}$ separates $H$ into two components $P_{1}$ and $P_{2}$ with $\partial P_{1}=T_{1} \cup S_{g, l}$ and $\partial P_{2}=T_{2} \cup S_{g, l}$, where $T_{1} \cup T_{2}=\partial H$ and $\partial T_{1}=\partial T_{2}=\partial S_{g, l}$.

Lemma 3.4. $T_{1}$ and $T_{2}$ are incompressible in $H$.

Proof. We have $H_{1}(H)=\mathbb{Z}+\mathbb{Z}+\mathbb{Z}$, with the three generators $y_{1}, y_{2}$ and $y_{3}$. By the preceding argument, $i_{*}\left(H_{1}\left(S_{g, l}\right)\right)$ is a subgroup of $H_{1}(H)$ generated by $2 y_{1}$, $2 y_{2}$ and $2 l y_{3}$. Thus $H_{1}(H) / i_{*}\left(H_{1}\left(S_{g, l}\right)\right)=\mathbb{Z}_{2} \oplus \mathbb{Z}_{2} \oplus \mathbb{Z}_{2 l}$ is a finite group.

Suppose $T_{1}$ or $T_{2}$ is compressible. Then it bounds a compressing disk $D_{1}$ in $H$. Since $\partial D \cap \partial S_{g, l}=\varnothing$ and $S_{g, l}$ is incompressible in $H$, by a standard argument in 3-manifold topology, we may assume that $D_{1} \cap S_{g, l}=\varnothing$. Since $H$ is a handlebody, we may also assume that $D_{1}$ is nonseparating in $H$. Thus there are two properly embedded disks $D_{2}$ and $D_{3}$ in $H$ such that $\left\{D_{1}, D_{2}, D_{3}\right\}$ is a set of basis disks of $H$. Let $z_{1}, z_{2}, z_{3}$ be generators of $\pi_{1}(H)$ corresponding to $D_{1}, D_{2}, D_{3}$. Since $S_{g, l}$ misses $D_{1}$, we have $i_{*}\left(\pi_{1}\left(S_{g, l}\right)\right) \subset G$, where $G$ is a subgroup of $\pi_{1}(H)$ generated by $z_{2}$ and $z_{3}$. Then $H_{1}(H) / i_{*}\left(H_{1}\left(S_{g, l}\right)\right)$ is an infinite group, a contradiction.

Proof of Proposition 3.2. Since $H$ is a handlebody and $S_{g, l}$ is incompressible in $H$, $P_{1}$ and $P_{2}$ are handlebodies. By Lemmas 3.3, 3.4 and the Handle Addition Lemma [Jaco 1984], $\hat{S}_{g, l}$ is incompressible in $P_{i}\left(\partial S_{g, l}\right)$ for $i=1$, 2. Since $H\left(\partial S_{g, l}\right)=$ $P_{1}\left(\partial S_{g, l}\right) \cup_{\hat{S}_{g, l}} P_{2}\left(\partial S_{g, l}\right)$, the surface $\hat{S}_{g, l}$ is incompressible in $H\left(\partial S_{g, l}\right)$.

\section{4. $H_{k}$ is irreducible, $\partial$-irreducible and anannular}

By construction, $K$ is cut by $E_{1} \cup E_{2}$ into ten $\operatorname{arcs} a_{1}, \ldots, a_{10}$, where $a_{i}$ arises from pushing $c_{i}$ into $\stackrel{\circ}{H}$. Now let $N(K)=K \times D$ be a regular neighborhood of $K$ in $H$, where the product structure has been adjusted so that $\bigcup_{i=1}^{10} \partial a_{i} \times D$ is contained in $E_{1} \cup E_{2}$. Let $H_{K}=H-\stackrel{\circ}{N}(K)$ and $F_{i}=E_{i}-\stackrel{\circ}{N}(K)$; also set $M_{i}=H_{K} \cap J_{i}$, for $i=1,2,3$, and $T=\partial(K \times D)$. Then $F_{1} \cup F_{2}$ separates $T$ into ten annuli $A_{1}, \ldots, A_{10}$ such that $A_{i}=a_{i} \times \partial D$.

$K$ and $C$ bound a nonembedded annulus $A_{*}$, which is cut by $E_{1} \cup E_{2}$ into ten disk $D_{1 *}, \ldots, D_{10 *}$ in $H$. Note that $D_{*}=\bigcup_{i \neq 6} D_{i *}$ is still a disk. Let $D_{i}=D_{i *} \cap H_{K}$ for $i \neq 6$. Then $D_{i}$ is a proper disk in some $M_{l}$ and $\bigcup_{i \neq 6} D_{i}$ is still a disk; see Lemma 4.1. Now we number the $\partial A_{i}$ such that $\partial_{1} A_{i}=\partial_{2} A_{i-1}$ and $\partial_{2} A_{i}=\partial_{1} A_{i+1}$. For $i \neq 6$, let $W_{i}=\overline{\partial N\left(D_{i} \cup A_{i}\right)-\partial M_{l}}$. Then $W_{i}$ is a proper separating disk in $M_{l}$. Each $W_{i}$ intersects $F_{1} \cup F_{2}$ in two $\operatorname{arcs} l_{i}$ and $l_{i+1}$. Note that $W=\bigcup_{i \neq 6} W_{i}$ is a disk. Thus $\partial W$ is a union of two arcs in $\partial H$ and $l_{6} \cup l_{7}$; see Figure 4. Since $c_{3}, c_{9}$ are parallel in $\partial J_{1}-\stackrel{\circ}{E}_{1}$, there are two arcs parallel to $c_{3}$ in $\partial J_{1}-\stackrel{\circ}{E}_{1}$, say $l^{\prime}, l^{\prime \prime}$, and two arcs in $F_{1}$, say $l^{1}, l^{2}$, such that $l^{\prime} \cup l^{\prime \prime} \cup l^{1} \cup l^{2}$ bounds a disk $W^{\prime}$ that separates $M_{1}$ into two handlebodies of genus two $H^{1}, H^{2}$ with $A_{1} \subset H^{1}$ and $A_{3}, A_{9} \subset H^{2}$. We denote by $\mu$ the meridian slope on $T$ and by $\tau$ the longitude slope on $T$.

We list some elementary facts about $K$ and $a_{i}$ : 


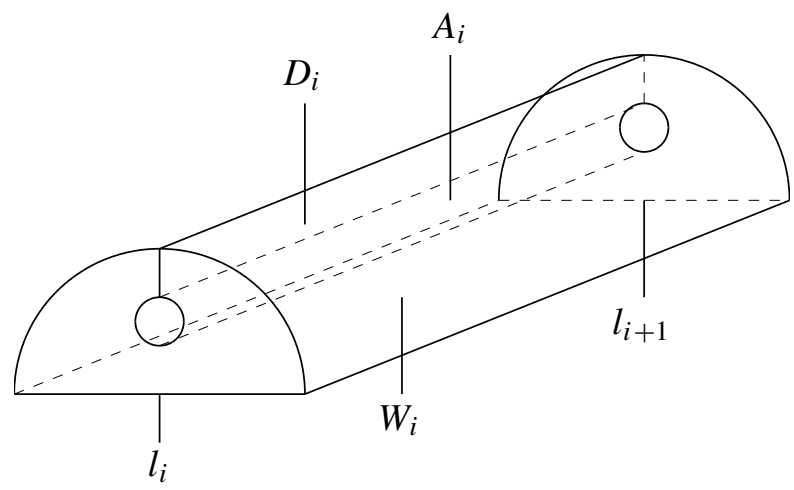

Figure 4

Lemma 4.0. (1) $K \neq 1$ in $\pi_{1}(H)$.

(2) Suppose $a_{i} \subset J_{m}$, where $i \neq 4,8$. Let $b_{i} \subset E_{1} \cup E_{2}$ be a given arc with $\partial b_{i}=\partial a_{i}$ and let $B \subset J_{m}$ be a nonseparating proper disk. Then $a_{i} \cup b_{i}$ intersects $\partial B$ in at least one point for all $i$, in at least three points when $i=7$, and in at least two points when $i=1,6$.

(3) There is no relative homotopy on $\left(J_{m}, E_{1} \cup E_{2}\right)$ sending $a_{i}$ to $E_{1} \cup E_{2}$.

Recall that a 3-manifold $M$ is irreducible if it contains no essential 2-spheres. $M$ is $\partial$-irreducible if $\partial M$ is incompressible. $M$ is atoroidal if it contains no essential tori. $M$ is anannular if it contains no essential annuli.

Lemma 4.1. $H_{K}$ is irreducible.

Proof. Suppose that $H_{K}$ is reducible, so there is an essential 2-sphere $S$ in $H_{K}$. Since $H$ is irreducible, $S$ bounds a 3-ball $B^{3}$ in $H$ and $K \subset B^{3}$, which contradicts Lemma 4.0(1).

Recall that $F$ is $\partial$-compressible if there is an essential arc $a$ in $F$ which, together with an $\operatorname{arc} b$ in $\partial M$, bounds a disk $D$ in $M$ such that $D \cap F=a$; otherwise, $F$ is $\partial$-incompressible.

Lemma 4.2. $F_{1} \cup F_{2}$ is incompressible and $\partial$-incompressible in $H_{K}$.

Proof. Suppose first that $F_{1} \cup F_{2}$ is compressible in $H_{K}$. Then there is a disk $B$ in $M$ such that $B \cap\left(F_{1} \cup F_{2}\right)=\partial B$ and $\partial B$ is an essential circle on $F_{1} \cup F_{2}$. Without loss of generality, we assume that $\partial B \subset F_{1}$ and $B \subset M_{2}$. Denote by $B^{\prime}$ the disk bounded by $\partial B$ in $E_{1}$. Then $B \cup B^{\prime}$ is a 2-sphere $S \subset J_{2}$, and it follows easily from Lemma 4.1 that $S$ bounds a 3-ball $B^{3}$ in $J_{2}$. Since $\partial B$ is essential in $F_{1}, B^{\prime}$ contains at least one component of $\partial a_{i}$. Since $S$ is separating and $a_{i}$ is connected, we must have $\left(a_{i}, \partial a_{i}\right) \subset\left(B^{3}, B^{\prime}\right)$, which provides a relative homotopy on $\left(J_{2}, E_{1}\right)$ sending $a_{i}$ to $E_{1}$. This contradicts Lemma 4.0(2). 
Now suppose $F_{1} \cup F_{2}$ is $\partial$-compressible in $H$. There is an essential arc $a$ in $F_{1} \cup F_{2}$ which, with an $\operatorname{arc} b$ in $\partial H_{k}$, bounds a disk $B$ in $H_{K}$ with $B \cap\left(F_{1} \cup F_{2}\right)=a$. Without loss of generality, we assume that $a \subset F_{2}$ and $B \subset M_{2}$. There are two cases: $b \subset T$. Then $b$ is a proper arc in one of $A_{4}, A_{6}$, and $A_{8}$, say $A_{6}$. If $b$ is not essential in $A_{6}$, then $a$ and an arc $b^{\prime}$ in $\partial A_{6}$ form an essential circle in $F_{2}$ bounding a disc in $M_{2}$. This contradicts the incompressibility of $F_{2}$ we just proved. If $b$ is essential in $A_{6}$, the disk $B$ provides a relative homotopy on $\left(J_{2}, E_{2}\right)$ sending $a_{6}$ to $E_{2}$, which contradicts Lemma 4.0(2).

$b \subset \partial H$. If $B$ is nonseparating in $J_{2}$, then $b_{6}$ can be chosen so that $a_{6} \cup b_{6}$ intersects $\partial B$ in at most one point, where $b_{6}$ is an arc in $E_{2}$ connecting the endpoints of $a_{6}$; this contradicts Lemma 4.0(2). If $B$ is separating in $J_{2}$, then $B$ separates $J_{2}$ into a 3-ball $B^{3}$ and a solid torus $J$. We denote by $D_{1}, D_{2}$ the two components of $E_{2}-a$. Since $a$ is essential in $F_{2}$, each of $\stackrel{\circ}{D}_{1}$ and $\stackrel{\circ}{D}_{2}$ contains at least one endpoint of $a_{4}$, $a_{6}$ and $a_{8}$.

Suppose that $D_{1} \subset B^{3}$ and $D_{2} \cup E_{1} \subset J$. By construction, $\partial_{1} a_{4}, \partial_{1} a_{8} \subset E_{1}$, $\partial_{2} a_{4}, \partial_{2} a_{8} \subset E_{2}$, and $\partial a_{6} \subset E_{2}$. Since $a_{4}, a_{6}$ and $a_{8}$ are disjoint from $B$, we have $a_{4}, a_{8} \subset J$ and $a_{6} \subset B^{3}$. This contradicts Lemma 4.0(2).

Suppose that $D_{1} \subset J$ and $D_{2} \cup E_{1} \subset B^{3}$. Then $a_{2}, a_{10} \subset B^{3}$. This contradicts Lemma 4.0(2).

\section{Lemma 4.3. $H_{K}$ is $\partial$-irreducible.}

Proof. Suppose $H_{K}$ is $\partial$-reducible. Let $B$ be a compressing disk of $\partial H_{K}$. If $\partial B \subset$ $T$, then $H_{K}$ contains an essential 2-sphere, which contradicts Lemma 4.1. Below we assume that $\partial B \subset \partial H$. Since $F_{1} \cup F_{2}$ is incompressible and $\partial$-incompressible in $H_{K}$ (Lemma 4.2), by a standard cut and paste argument, we may assume that $B \cap\left(F_{1} \cup F_{2}\right)=\varnothing$. We assume that $B \subset M_{2}$. (The other cases are similar.) Then $B$ misses $b_{6}$. If $B$ is nonseparating in $J_{2}$, by Lemma 4.0(2), $B$ intersects $a_{6}$, a contradiction. If $B$ is separating, then $B$ separates a 3-ball $B^{3}$ from $J_{2}$. Since $\partial B$ is essential in $\partial H_{K}$, there are two cases: Either $B^{3}$ contains only one of $E_{1}$ and $E_{2}$, say $E_{1}$, in which case $a_{8} \cap B \neq \varnothing$, a contradiction; or $B^{3}$ contains both $E_{1}$ and $E_{2}$, in which case there is a relative homotopy on $\left(J_{2}, E_{2}\right)$ sending $a_{6}$ to $E_{2}$, in contradiction with Lemma 4.0(2).

\section{Lemma 4.4. $M$ is anannular.}

Proof. Suppose $H_{K}$ contains an essential annulus $A$. We can choose $A$ so that $\left|A \cap\left(F_{1} \cup F_{2}\right)\right|$ is minimal among all essential annuli in $H_{K}$. This condition, together with Lemma 4.2 and the proof of Lemma 4.3, implies that each component of $A \cap\left(F_{1} \cup F_{2}\right)$ is essential in both $A$ and $F_{1} \cup F_{2}$. There are three cases:

Case 1: $\partial A \subset T$. Here $A$ is separating in $H_{k}$; otherwise, $H$ contains either a nonseparating 2-sphere or a nonseparating torus. Hence the union of $A$ and an 
annulus $A^{\prime}$ on $T$ makes a separating torus $T^{\prime}$, cutting off a manifold with boundary $T \cup T^{\prime}$. Since $M$ is irreducible, $T^{\prime}$ is incompressible, so by Lemma $5.5 T^{\prime}$ is parallel to $T$, which implies that $A$ is inessential. (The arguments in Section 5 are independent of those in Section 4.)

Case 2: $\partial_{1} A \subset T$ and $\partial_{2} A \subset \partial H$. By Lemma 4.3, both $\partial H$ and $T$ are incompressible in $H_{K}$. Clearly $H_{K}$ is not homeomorphic to $T \times I$. Since Dehn fillings along $\mu$ and $\partial A_{1}$ both compress $\partial H$, by an important theorem in Dehn filling, $\Delta\left(\partial_{1} A, \mu\right) \leq 1$. See [Culler et al. 1987, 2.4.3].

We first suppose that $\partial_{1} A$ is the meridian slope $\mu$. Then $\partial_{1} A$ is disjoint from $F_{1} \cup F_{2}$. We claim that $A$ is disjoint from $F_{1} \cup F_{2}$.

Suppose, to the contrary, that $A \cap\left(F_{1} \cup F_{2}\right) \neq \varnothing$. Since $F_{1} \cup F_{2}$ is incompressible and $\partial$-incompressible in $H_{K}$ (Lemma 4.2), by a standard cut and paste argument, we may assume that $\partial_{2} A \cap\left(F_{1} \cup F_{2}\right)=\varnothing$. Now each component of $A \cap\left(F_{1} \cup F_{2}\right)$ is an essential simple closed curve in $A$. Let $a$ be an outermost circle in $A \cap\left(F_{1} \cup F_{2}\right)$. Then $a$ and $\partial_{1} A$ bound an annulus $A^{*}$ in $A$ such that $\AA^{*}$ is disjoint from $F_{1} \cup F_{2}$. We may assume that $a \subset F_{1}$ and $\partial_{1} A \subset A_{i}$ for some $i$. Let $B^{*}$ be the disk bounded by $a$ on $E_{1}$ and let $D$ be the meridian disk of $N(K)$ bounded by $\partial_{1} A$. Since $a$ is essential on $F_{1}, B^{*}$ contains at least one component of $\partial F_{1}$. In $H, B^{*} \cup A^{*} \cup D$ is a separating 2-sphere $S^{2}$ that bounds a 3-ball $B^{3}$. For $j \neq i$, if $\partial_{1} a_{j} \subset B^{*}$, then $\partial_{2} a_{j} \subset B^{*}$ and $a_{j} \subset B^{3}$. This possibility is ruled out by Lemma 4.0(2). Note also that $\partial_{1} a_{i} \subset B^{*}$ and that $\partial_{2} a_{i}$ is not contained in $B^{*}$. Now let $A^{\prime}$ be the annulus bounded by $a$ and $\partial_{1} a_{i} \times \partial D=\partial_{1} A_{i}$ in $F_{1}$. Then $A^{*} \cup A^{\prime}$ is isotopic to an annulus disjoint from $F_{1} \cup F_{2}$. By the preceding argument, $A^{*} \cup A^{\prime}$ is inessential. Thus we can properly isotope $A$ by pushing the annulus $A^{*}$ to the other side of $F_{1}$ to reduce $\left|A \cap\left(F_{1} \cup F_{2}\right)\right|$, contradicting our choice of $A$ at the beginning of the proof.

We may assume that $A$ is contained in $M_{2}$. Let $D$ be the meridian disk of $N(K)$ bounded by $\partial_{1} A$ and set $B=A \cup_{\partial_{1} A} D$. Then $B$ is a proper disk in $J_{2}$, meeting $K$ in exactly one point; hence $B$ is a meridian disk of $J_{2}$. Let $b_{6}$ be an arc on $E_{2}$ connecting the two endpoints of $c_{6}$. Then $c_{6} \cup b_{6}$ would be a closed curve of winding number 2 in the solid torus $J_{2}$ intersecting $B$ at most once, which is absurd.

Next we suppose that $\Delta\left(\partial_{1} A, \mu\right)=1$. Then $A$ is cut by $\left(F_{1} \cup F_{2}\right)$ into ten squares $S_{i}, i=1, \ldots, 10$, each of which has two opposite sides in $F_{1} \cup F_{2}$, the other two sides being the longitude arc $a_{i}$ in $A_{i}$ and $a_{i}^{*} \subset \partial H$. Let $b_{2}^{*}$ be the arc connecting the two endpoints of $a_{2}^{*}$ in $E_{1}$ and let $b_{6}^{*}$ be the arc connecting the two endpoints of $a_{6}^{*}$ in $E_{2}$. The two simple closed curves $b_{2}^{*} \cup a_{2}^{*}$ and $b_{6}^{*} \cup a_{6}^{*}$ on $\partial J_{2}$ are disjoint. But in $\pi_{1}\left(J_{2}\right)$, we have $b_{2}^{*} \cup a_{2}^{*}=y_{2}$ and $b_{6}^{*} \cup a_{6}^{*}=y_{2}^{2}$, a contradiction.

Case 3: $\partial A \subset \partial H$. Suppose first that $A \cap\left(F_{1} \cup F_{2}\right)=\varnothing$. Then $A$ is contained in one of $M_{1}, M_{2}$ and $M_{3}$. Since $A$ is essential and $H_{K}$ is $\partial$-irreducible, $A$ is disjoint from 
$D_{i}$ for $i \neq 6$. Since each component of $\partial H \cap J_{1}-c_{1} \cup c_{3}$ and $\partial H \cap J_{3}-c_{5} \cup c_{7}$ is a disc, $A \subset M_{2}$. Since $A$ is disjoint from $c_{2}, c_{4}, c_{8}, c_{10}$, each component of $\partial A$ intersects $B_{2}$ in only one point in $J_{2}$ (see Figure 2). Thus $A$ is isotopic to each component of $\partial J_{2}-\partial A$ in $J_{2}$. This means that $A$ is not essential in $M_{2}$, a contradiction.

Now suppose that $A \cap\left(F_{1} \cup F_{2}\right) \neq \varnothing$. There are two subcases:

Case 3a: Each component of $A \cap\left(F_{1} \cup F_{2}\right)$ is an essential circle. Let $a$ be an outermost component of $A \cap\left(F_{1} \cup F_{2}\right)$. That means that $\partial_{1} A$, together with $a$, bounds an annulus $A^{*}$ in $A$ such that $A^{*} \cap\left(F_{1} \cup F_{2}\right)=a$. Then $A^{*} \subset M_{i}$. We denote by $B^{*}$ the disk bounded by $a$ in $E_{1} \cup E_{2}$. Let $D^{*}=A^{*} \cup B^{*}$. Then $D^{*}$ is a disk. Let $D$ be the disk obtained from $D^{*}$ by pushing $B^{*}$ slightly into $J_{l}$. Then $D$ is a properly embedding disk in $J_{l}$ such that $D$ intersects each $a_{i}$ in at most two points. Furthermore, if $D$ intersects $a_{i}$ in two points for some $i$, the two endpoints of $a_{i}$ lie in $B^{*}$. Thus, in this case, the algebraic intersection number of $a_{i}$ and $D$ is 0 . By Lemma 4.0, $A^{*}$ is separating in $J_{l}$.

Suppose that $A^{*}$ is contained in one of $J_{1}$ and $J_{3}$, say $J_{1}$. Then $\partial_{1} A$ is parallel to $\partial E_{1}$. We denote by $A^{\prime}$ the annulus bounded by $\partial_{1} A$ and $a$ in $\partial J_{1}$. Since $a$ is essential in $F_{1}, B_{*}$ contains at least one endpoint of $a_{1}, a_{3}, a_{9}$. Furthermore, $\partial_{1} a_{i} \subset B^{*}$ if and only if $\partial_{2} a_{i} \subset B^{*}$. Now if $\partial_{1} a_{j} \subset A^{\prime}$ for some $j$, then $\partial_{2} a_{j} \subset A^{\prime}$. This means that $a_{j}$ is disjoint from $B_{1}$ as in Figure 1, a contradiction. Thus for each $i, j$, we have $\partial_{j} a_{i} \subset B^{*}$, which means that $a$ is parallel to $\partial E_{1}$ in $F_{1}$. Now $\partial D_{i}$, for $i=1,3,9$, intersects each component of $\partial A^{*}$ in two points, which means that $D_{i}$ intersects $A^{*}$ in two arcs each of which has its two endpoints in distinct components of $\partial A^{*}$. (Otherwise, since $\partial_{1} A$ is isotopic to $\partial E_{1}$, we would have $a_{i} \cup b_{i}=1$ in $\pi_{1}\left(J_{1}\right)$, where $b_{i}$ is an arc in $\partial E_{1}$ connecting the two endpoints of $a_{i}$, a contradiction.) Thus we can push $\partial_{1} A$ into $M_{2}$ to reduce $\left|A \cap\left(F_{1} \cup F_{2}\right)\right|$, contradicting our assumption on $A$.

Suppose instead that $A^{*} \subset M_{2}$. Without loss of generality, we assume that $a \subset F_{1}$. We denote by $A^{\prime}$ the annulus bounded by $\partial E_{1}$ and $a$ in $E_{1}$. Then $A^{\prime}$ and $B^{*}$ lie on distinct sides of $J_{2}-A^{*}$. If $\partial_{1} A$ is isotopic to $\partial E_{2}$, then $a_{6} \cup b_{6}=1$ in $\pi_{1}\left(J_{2}\right)$ where $b_{6}$ is an arc in $E_{2}$ connecting the two endpoints of $a_{6}$, a contradiction. If $\partial_{1} A$ bounds a disk $D$ in $\partial J_{2}$ such that $E_{1}, E_{2} \subset D$, then $a_{4} \cup a_{8} \cup b^{1} \cup b^{2}=1$ in $\pi_{1}\left(J_{2}\right)$, where $b^{i}$ is an arc in $E_{i}$ connecting the endpoints of $a_{4 i}$ and $a_{8}$, a contradiction. Now $\partial_{1} A$ is isotopic to $\partial E_{1}$. Then $D_{4}$ intersects $A^{*}$ in an arc. By the preceding argument, we can push $\partial_{1} A$ into $M_{1}$ to reduce $\left|A \cap\left(F_{1} \cup F_{2}\right)\right|$.

Case 3b: Each component of $A \cap\left(F_{1} \cup F_{2}\right)$ is an essential arc. Then $F_{1} \cup F_{2}$ cuts $A$ into proper squares $S_{i}$ in $M_{l} \subset J_{l}$, each $S_{i}$ having two opposite sides in $F_{1} \cup F_{2}$ and the remaining two sides in $\partial H$. If $S_{i} \subset J_{l}$ for $l=2$ or 3 , then $S_{i}$ is a separating disc in $J_{l}$. Otherwise, say $S_{i}$ is a nonseparating disc in $J_{2}$. By the same reason as that at the end of the proof of Lemma 4.3, the fact that $S \cap\left(F_{1} \cup F_{2}\right)$ consists of 


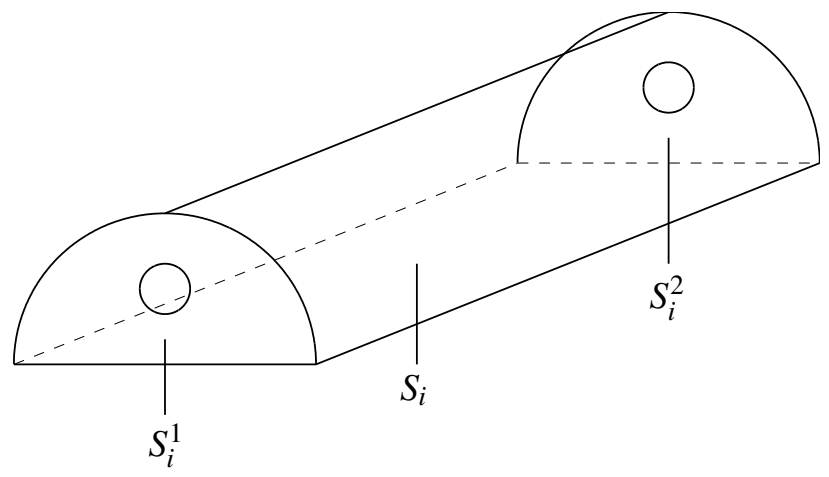

Figure 5

two proper arcs in $E_{1} \cup E_{2}$ implies that $b_{6}$ can be chosen so as to intersect $\partial S_{i}$ in at most two points; furthermore, if $b_{6}$ intersects $\partial S_{i}$ in two points then $S_{i} \cap F_{1}=\varnothing$ and $S_{i} \cap b_{2}=\varnothing$, where $b_{i}$ is an arc in $E_{1} \cup E_{2}$ connecting the two endpoints of $a_{i}$. This means that $S_{i}$ meets $a_{2}$ or $a_{6}$ by Lemma 4.0(1), a contradiction. Now each $S_{i}$ cuts off a 3-ball $B_{i}^{3}$ from $J_{l}$ for $l=2$ or 3 as in Figure 5. Let $S_{i}^{1}$ and $S_{i}^{2}$ be the two disks of $B_{i}^{3} \cap\left(E_{1} \cup E_{2}\right)$ and $S_{i} \subset J_{l}$ where $l=2$ or 3. By Lemma 4.0(2), we have:

(i) $\partial_{1} a_{j} \subset S_{i}^{1}$ if and only if $\partial_{2} a_{j} \subset S_{i}^{2}$.

(ii) If $a_{j}$ is contained in $B_{i}^{3}$, then $a_{l}$ is not contained in $B_{i}^{3}$.

This means that for each $i$, there is only one boundary component of $F_{1} \cup F_{2}$ lying in each of $S_{i}^{1}$ and $S_{i}^{2}$. Thus if $S_{i}$ lies in $M_{1}$ for some $i$, then $S_{i}$ is also separating in $J_{1}$. Otherwise, say $S_{i}$ is nonseparating in $J_{1}$. By (i) and (ii), the three circles $a_{1} \cup b_{1}, a_{3} \cup b_{3}, a_{9} \cup b_{9}$ intersect $S_{i}$ in two points, a contradiction. It follows that $S_{i}$ is also as in Figure 5 and $A$ cuts off a solid torus $P$ from $H$. Thus $D_{i *}$ can be chosen to be disjoint from $A$ even if $i=6$. This means that $K$ and a component of $\partial A$ bound an annulus, which has been ruled out in Case 2 .

\section{5. $H_{K}$ contains no closed essential surface}

Suppose $H_{K}$ contains essential closed surfaces. Let $W, W^{\prime}$ and $W_{i}$ be the disks defined in Section 4. Denote by $X(F)$ the union of the components of $F \cap M_{1}$ isotopic to $\partial H \cap M_{1}$. We define the complexity on the essential closed surfaces $F$ in $H_{K}$ by the quadruple

$$
C(F)=\left(|F \cap W|,\left|F \cap F_{2}\right|,\left|\left(F \cap M_{1}-X(F)\right) \cap W^{\prime}\right|,\left|F \cap F_{1}\right|\right) .
$$

We rank complexities in lexicographic order. Suppose $F$ minimizes $C(F)$. By a standard argument in 3-manifold topology, we derive the following facts:

Lemma 5.0. (1) Each component of $F \cap\left(F_{1} \cup F_{2}\right)$ is an essential circle in both $F$ and $F_{1} \cup F_{2}$. 

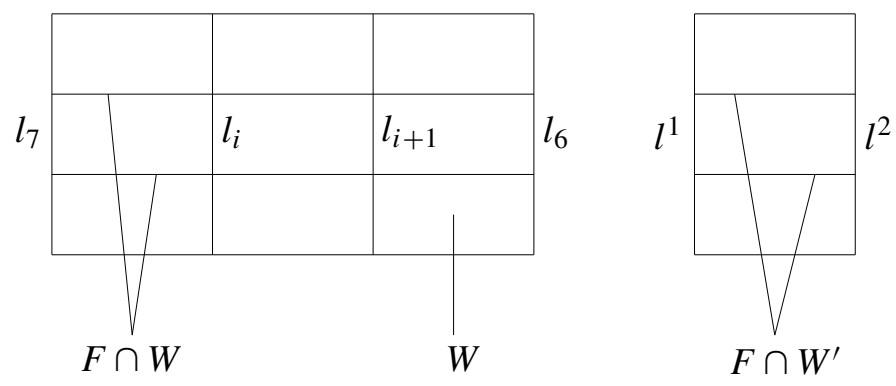

Figure 6

(2) Each component of $F \cap W$ is an arc in $W$ one of whose endpoints lies in $l_{6}$ and the other in $l_{7}$. Similarly each component of $F \cap W^{\prime}$ is an arc in $W^{\prime}$ one of whose endpoints lies in $l^{1}$ and the other in $l^{2}$. Hence $\left|F \cap l_{i}\right|=\left|F \cap l_{j}\right|$ for all $i, j$ and $\left|F \cap l^{1}\right|=\left|F \cap l^{2}\right|$ as in Figure 6.

(3) Each component of $F \cap\left(F_{1} \cup F_{2}\right)$ isotopic to $\partial A_{i}$ is disjoint from $W \cup W^{\prime}$.

For two surfaces $P_{1}$ and $P_{2}$ in a 3-manifold, a pattern of $P_{1} \cap P_{2}$ is a set of disjoint arcs and circles representing isotopy classes of $P_{1} \cap P_{2}$. For each isotopy class $s$, we denote by $v(s)$ the number of components of $P_{1} \cap P_{2}$ in the isotopy class $s$.

The proof of the next lemma is similar to that of [Qiu and Wang 2004, Lemma 4.3].

Lemma 5.1. Each component of $F \cap M_{3}$ is isotopic to one of $\partial H \cap M_{3}, A_{5}$ and $A_{7}$. Proof. The four arcs $l_{5}, l_{6}, l_{7}, l_{8}$ separate $F_{2}$ into four annuli $A^{5}, A^{6}, A^{7}, A^{8}$ and a disk $D$. By the minimality of $|F \cap W|$, the pattern of $F \cap A^{j}$ is as in Figure 7, left, and the pattern of $F \cap D$ is as in Figure 7, right. Since $\left|F \cap l_{i}\right|$ is a constant, $v\left(d_{5}\right)=0$. If $v\left(d_{i}\right) \neq 0$ for $1 \leq i \leq 4$, then $F \cap F_{2}$ contains $\min \left(v\left(d_{1}\right), \ldots, v\left(d_{4}\right)\right)$
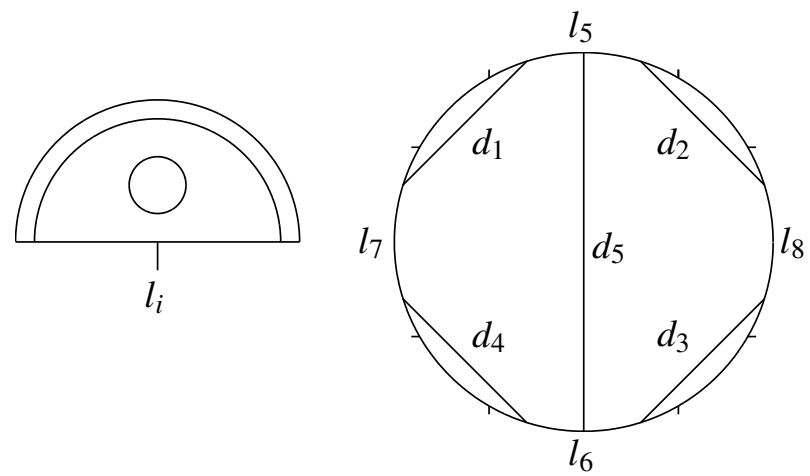

Figure 7 

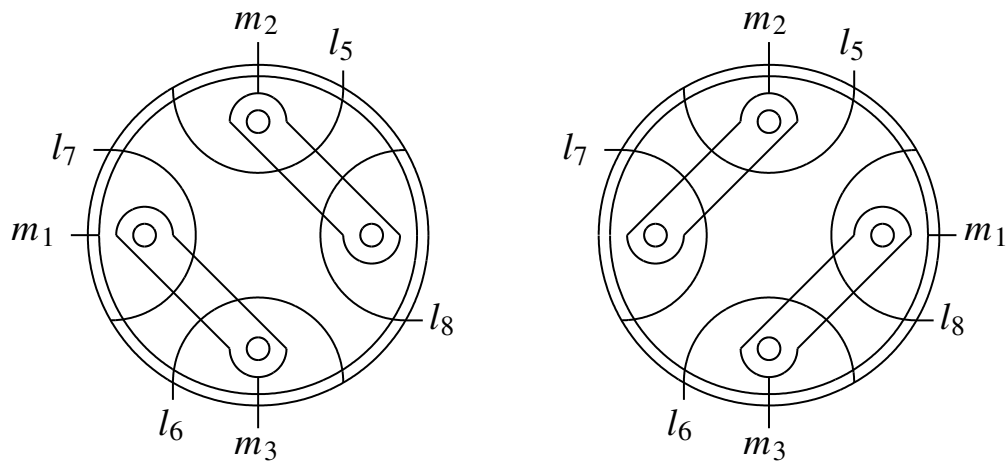

Figure 8

components parallel to a disk on $\partial E_{2}$. Now if $v\left(d_{1}\right)=0$, then $v\left(d_{3}\right)=0$. Similarly, if $v\left(d_{2}\right)=0$, then $v\left(d_{4}\right)=0$. Thus according to the order of $l_{5}, l_{6}, l_{7}, l_{8}$ in $F_{2}$, the pattern of $F \cap F_{2}$ is as in one of the diagrams in Figure 8, with $v\left(m_{2}\right)=v\left(m_{3}\right)$. Note that $W_{5}$ and $W_{7}$ separate $M_{3}$ into three solid tori $J^{1}, J^{2}, J^{3}$. Without loss of generality, we assume that $A_{5} \subset J^{1}, A_{7} \subset J^{2}$. Let $S=F \cap M_{3}$ and $S^{\prime}$ be a component of $S$.

Now we claim that if one of component of $\partial S^{\prime}$ is isotopic to $\partial E_{2}$, then $S^{\prime}$ is isotopic to $\partial H \cap M_{3}$.

Let $\partial_{1} S$ be the outermost component of $\partial S$ isotopic to $\partial E_{2}$. Now $\partial_{1} S$ intersects $l_{i}$ as in Figure 8. Without loss of generality, we assume that $\partial_{1} S \subset \partial S^{\prime}$. We denote by $e_{i}$ the arc $\partial_{1} S \cap A^{i}$. Now let $S_{l}=S^{\prime} \cap J^{l}$, then $S_{l}$ is an incompressible surface in $J^{l}$. Note that $\partial S_{1}=e_{5} \cup e_{6} \cup\left(S \cap W_{5}\right)$ bounds a disk in $J^{1}$ parallel to a disk on $\partial M_{3}$. Similarly $S_{2}$ is a disk in $J^{2}$ parallel to a disk on $\partial M_{3}$ bounded by $e_{7} \cup e_{8} \cup\left(S \cap W_{7}\right)$. $\partial S_{3}$ also has one component which is trivial in $\partial M_{3}$, as in Figure 9, left. Hence one component of $S_{3}$ is a disk in $J^{3}$ parallel to $\partial J^{3}$, Thus $S^{\prime}=S_{1} \cup_{S \cap W_{5}} S_{3} \cup_{S \cap W_{7}} S_{2}$ is isotopic to $M_{3} \cap \partial H$.

Now we claim that $v\left(m_{2}\right)=v\left(m_{3}\right)=0$ in both parts of Figure 8 .

Let $S_{0}=S-X^{\prime}$, where $X^{\prime}$ is a subset of $S$ each of whose components is isotopic to $\partial H \cap M_{3}$. Then no component of $\partial S_{0}$ is isotopic to $\partial E_{2}$. Let $P_{3}=S_{0} \cap J^{3}$. If
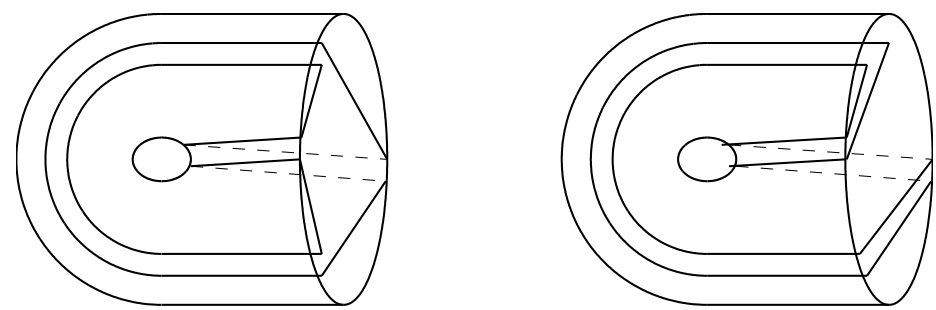

Figure 9 


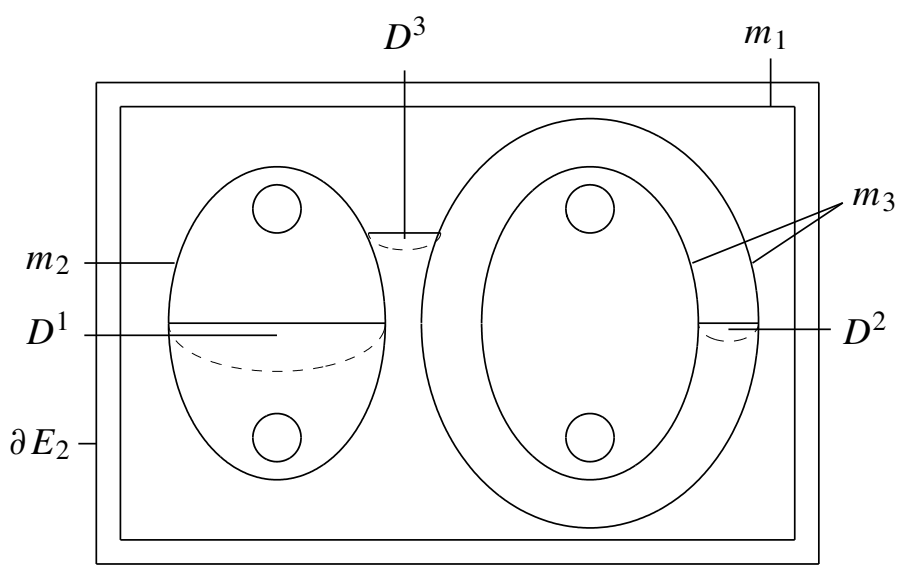

Figure 10

$v\left(m_{2}\right) \neq 0$, then $P_{3}$ is incompressible in $J^{3}$ and $\partial P_{3}$ contains $2 v\left(m_{2}\right)=2 v\left(m_{3}\right)$ components $c$, as in Figure 9, right. Since $a_{7}$ intersects a basis disk $B_{3}$ of $J_{3}$ in three points and $a_{5}$ intersects $B_{3}$ in one point, $c$ does not bound a disk in $J^{3}$. Since $J^{3}$ is a solid torus, each component of $P_{3}$ is a $\partial$-compressible annulus. Let $D^{*}$ be a $\partial$-compressing disk of an outermost component of $P_{3}$. This disk can be isotoped so that $D^{*} \cap \partial J^{3} \subset E_{2} \cap J^{3}$. Then, back in $J_{3}, D^{*}$ is isotopic to one of $D^{1}, D^{2}, D^{3}$ as in Figure 10. In the case of $D^{1}$ or $D^{2}$, one can push $F$ along the disc to reduce $|F \cap W|$; in the case of $D^{3}$, one can push $F$ along the disc to reduce $\left|F \cap F_{2}\right|$, without increasing $|F \cap W|$. Either way, the minimality of $C(F)$ is contradicted.

Now let $P$ be a component of $S=F \cap M_{3}$. If one component of $\partial P$ is isotopic to $\partial E_{2}$, then $P$ is isotopic to $M_{3} \cap \partial H$. If not, each component of $\partial P$ is isotopic to one component of $\partial A_{5} \cup \partial A_{7}$. By the minimality of $C(F), P$ is contained in $J^{1}$ or $J^{2}$. It is easy to see that $P$ is isotopic to one of $A_{5}$ and $A_{7}$.

Now we consider $S=F \cap M_{1}$. Note that $W_{1}$ and $W^{\prime}$ separate $M_{1}$ into two solid tori $J^{1}, J^{2}$ and a handlebody of genus two $H^{\prime}$ such that $A_{1} \subset J^{1}$ and $A_{3}, A_{9} \subset H^{\prime}$; moreover $l_{1}, l_{2}, l^{1}, l^{2}$ separate $F_{1}$ into two annuli and two planar surfaces with three boundary components and a disk $D$ such that $\partial J^{2} \cap F_{1}=D$. See Figure 11 . Let $k_{1}$ be a component of $F \cap W_{1}, k_{2}$ a component of $F \cap W^{\prime}$, and $k_{i}^{\prime}$, for $i=1,2$, an arc in $D$ connecting the two endpoints of $k_{i}$. Let $\alpha=k_{1} \cup k_{1}^{\prime}$ and $\beta=k_{2} \cup k_{2}^{\prime}$. Note that $k_{1}^{\prime}$ and $k_{2}^{\prime}$ can be chosen so that $\beta$ intersects $\alpha$ in one point. Furthermore, by construction, $\alpha$ intersects a basis disk of $J^{2}$ in two points and $\beta$ intersects a basis disk of $J^{2}$ in one point. Now we fix the orientations of $\alpha$ and $\beta$ so that $\alpha=y^{2}$ and $\beta=y$, where $y$ is a generator of $\pi_{1}\left(J^{2}\right)$. Then $\alpha \beta^{-2}$ is an essential circle in $\partial J^{2}$ and null homotopic in $J^{2}$.

The next lemma follows immediately from the proof of Lemma 5.1. 

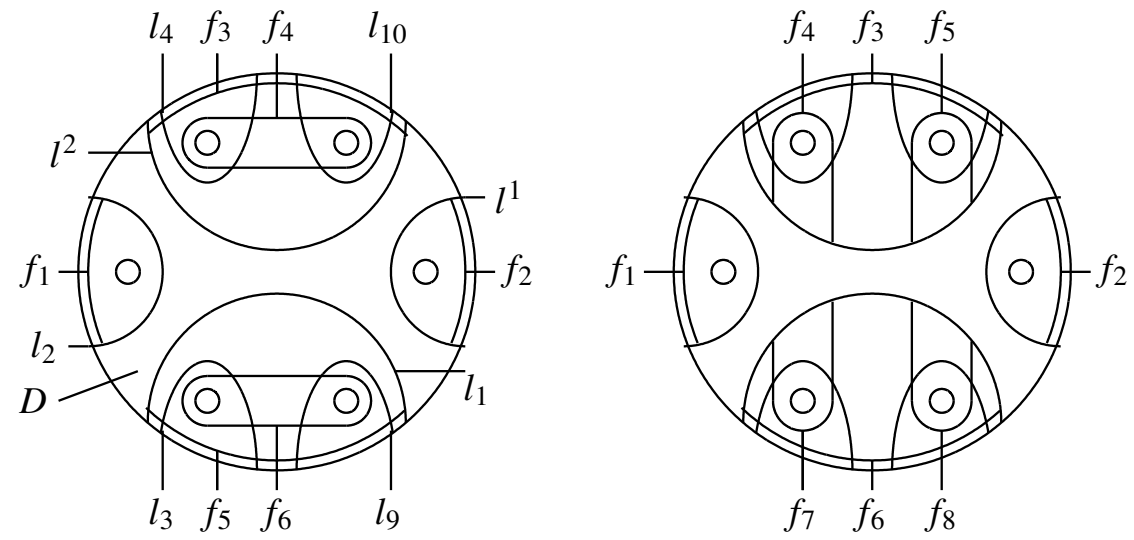

Figure 11

Lemma 5.2. Let $P$ be a component of $S=F \cap M_{1}$. If one component of $\partial P$ is isotopic to $\partial E_{1}$. Then $P$ is isotopic to $M_{1} \cap \partial H$.

By the construction and Lemma 5.0, the pattern of $\partial S \cap\left(F_{1} \cap\left(J^{1} \cup H^{\prime}\right)\right)$ is as in one of the diagrams in Figure 11, and moreover

(1) in Figure 11, left, we have $v\left(f_{1}\right)=v\left(f_{2}\right), v\left(f_{3}\right)=v\left(f_{5}\right), v\left(f_{4}\right)=v\left(f_{6}\right)$ and $v\left(f_{3}\right)+v\left(f_{4}\right)=v\left(f_{1}\right) ;$

(2) in Figure 11, right, we have $v\left(f_{1}\right)=v\left(f_{2}\right)=v\left(f_{3}\right)+v\left(f_{4}\right), v\left(f_{3}\right)=v\left(f_{6}\right)$ and $v\left(f_{4}\right)=v\left(f_{5}\right)=v\left(f_{7}\right)=v\left(f_{8}\right) \neq 0$.

Lemma 5.3. If the pattern of $S \cap\left(F_{1} \cap\left(J^{1} \cup H^{\prime}\right)\right)$ is as in Figure 11, left, the pattern of $S \cap F_{1}$ is as in Figure 12 with $v\left(n_{2}\right)=v\left(n_{3}\right)=v\left(n_{4}\right)$.

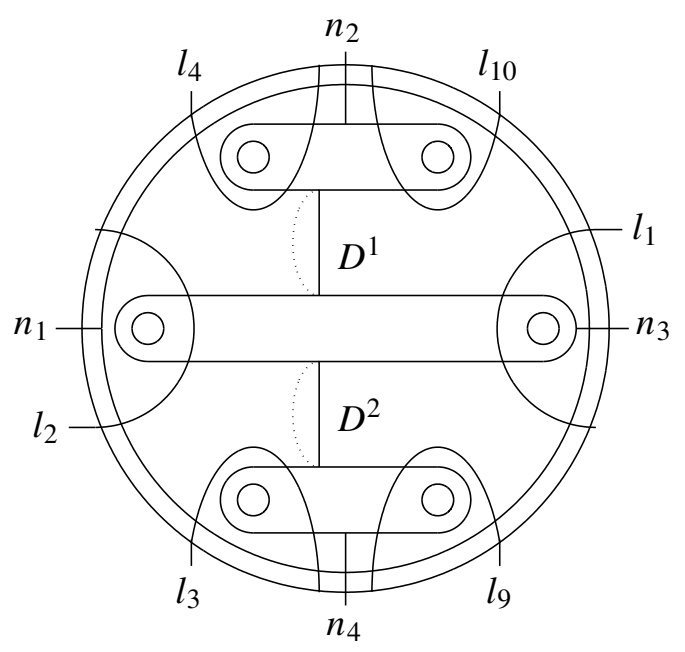

Figure 12 


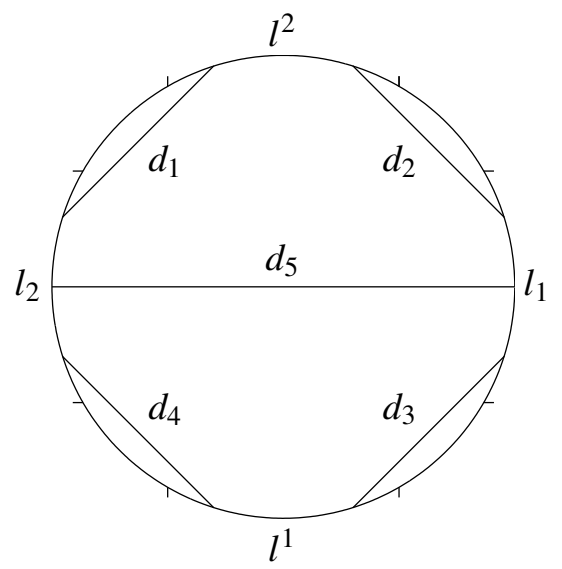

Figure 13

Proof. If $v\left(f_{3}\right)=0$, the pattern of $S \cap F_{1}$ is as in Figure 12 with $v\left(n_{2}\right)=v\left(n_{3}\right)=$ $v\left(n_{4}\right)$ and $v\left(n_{1}\right)=0$.

Suppose instead that $v\left(f_{3}\right) \neq 0$. Since $v\left(f_{3}\right)=v\left(f_{5}\right) \leq v\left(f_{1}\right)=v\left(f_{2}\right)$, the pattern of $S \cap D$ is as in Figure 13, where $v\left(d_{1}\right)=v\left(d_{3}\right)$ and $v\left(d_{2}\right)=v\left(d_{4}\right)$. If $v\left(d_{1}\right), v\left(d_{2}\right) \neq$ 0 , then $S \cap F_{1}$ contains $\min \left(v\left(d_{1}\right), v\left(d_{2}\right)\right)$ components isotopic to $\partial E_{1}$. Thus if $v\left(d_{1}\right)=v\left(d_{2}\right)$, then $S \cap F_{1}$ is as in Figure 12 with $v\left(n_{2}\right)=v\left(n_{3}\right)=v\left(n_{4}\right)$. Now without loss of generality, we assume that $v\left(d_{1}\right)<v\left(d_{2}\right)$. Let $k=v\left(d_{2}\right)-v\left(d_{1}\right)$. By Lemmas 5.0(2) and 5.2, $\partial\left(S \cap J^{2}\right)$ contains $n=\operatorname{gcd}\left(k, k+v\left(d_{5}\right)\right)$ components $c$ isotopic to $\alpha^{p} \beta^{q}$, where $|p|=\left(k+v\left(d_{5}\right)\right) / n$ and $|q|=k / n$. Since $y+v\left(d_{5}\right) \geq y, c$ is not null homotopic in $J^{2}$. Moreover, $c$ intersects both $d_{2}$ and $d_{4}$; if $v\left(d_{5}\right) \neq 0$, then $c$ also intersects $d_{5}$. Thus these curves separate $\partial J^{2}$ into $m$ annuli $A^{1}, \ldots, A^{m}$ such that, for each $j$, there is an arc in $D \cap A^{j}$ connecting the two boundary components of $A^{j}$. Since $J^{2}$ is a solid torus, each component of $(S-X(F)) \cap J^{2}$ is an annulus. Let $D^{*}$ be a $\partial$-compressing disk of $(S-X(F)) \cap J^{2}$. Then $D^{*}$ can be moved so that $D^{*} \cap \partial J^{2}=D^{*} \cap D=a$. Thus there are three possibilities:

1. The two endpoints of $a$ lie in one of $d_{2}, d_{4}, d_{5}$. Then $D^{*}$ is one of $D^{1}, D^{3}$ as in Figure 14, left. In each case, one can push $F$ along the disc to reduce $|F \cap W|$, a contradiction.

2. One endpoint of $a$ lies in $d_{2} \cup d_{4}$ and the other lies in $d_{5}$. Then $D^{*}$ is $D^{2}$ as in Figure 14, left. This case is similar to the previous case.

3. One endpoint of $a$ lies in $d_{2}$ and the other lies in $d_{4}$. In this case, $v\left(d_{5}\right)=0$. By Lemma 5.0(2), we have $v\left(f_{4}\right)=v\left(f_{6}\right)=0$ in Figure 11, left. Now the pattern of $S \cap F_{1}$ is as in Figure 14, right, and $D^{*}$ is also as in the same figure. By doing a surgery on $F$ along $D^{*}$, we obtain a surface $F^{\prime}$ isotopic to $F$ such that $\left|F^{\prime} \cap W\right|=$ $|F \cap W|,\left|F^{\prime} \cap F_{2}\right|=\left|F \cap F_{2}\right|$ and $\left|\left(F^{\prime} \cap M_{1}-X\left(F^{\prime}\right)\right) \cap W^{\prime}\right|<\left|\left(F \cap M_{1}-X(F)\right) \cap W^{\prime}\right|$ (by Lemma 5.2), contradicting minimality. 

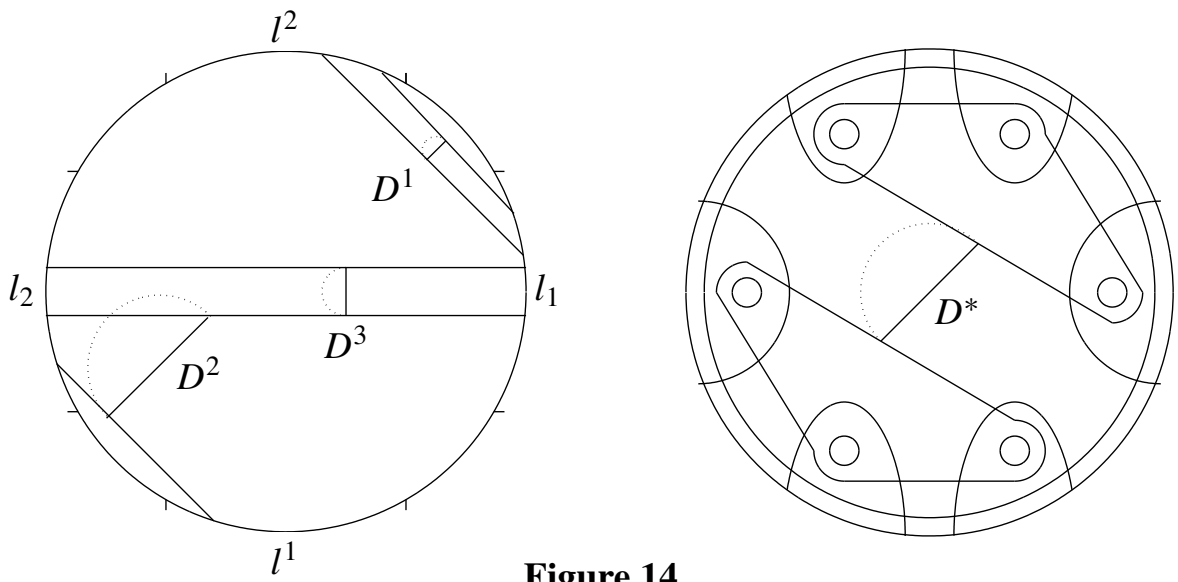

Figure 14

Lemma 5.4. If the pattern of $S \cap\left(F_{1} \cap\left(J_{1} \cup H^{\prime}\right)\right)$ is as in Figure 11, right, then the pattern of $S \cap F_{1}$ is as in Figure 15.

Proof. We have $v\left(f_{1}\right)=v\left(f_{2}\right)=v\left(f_{3}\right)+v\left(f_{4}\right)=v\left(f_{6}\right)+v\left(f_{7}\right)$. Thus the pattern of $S \cap D$ is as in Figure 16, where $v\left(d_{1}\right)=v\left(d_{3}\right), v\left(d_{2}\right)=v\left(d_{4}\right)$, and $v\left(d_{5}\right)=2 v\left(f_{5}\right)$. Therefore $v\left(d_{5}\right) \neq 0$. Referring to Figure 11, right, we distinguish two cases: $v\left(f_{3}\right)=v\left(f_{6}\right)=0$ and $v\left(f_{3}\right)=v\left(f_{6}\right)=0$.

If $v\left(f_{3}\right)=v\left(f_{6}\right)=0$, we have $v\left(d_{5}\right)=v\left(d_{1}\right)+v\left(d_{2}\right)$. There are three subcases:

Suppose first that $v\left(d_{1}\right)=v\left(d_{2}\right)$. Since $v\left(d_{5}\right) \neq 0, \partial\left(S \cap J^{2}\right)$ contains $v\left(d_{1}\right)$ trivial components in $\partial J^{2}$ bounding some disks in $S$ as in Figure 9, left, and $v\left(d_{5}\right)$ components isotopic to $\beta$. Since $\beta$ intersects a basis disk of $J^{2}$ in one point, each nontrivial component of $S \cap J^{2}$, say $A^{*}$, is an annulus parallel to each component

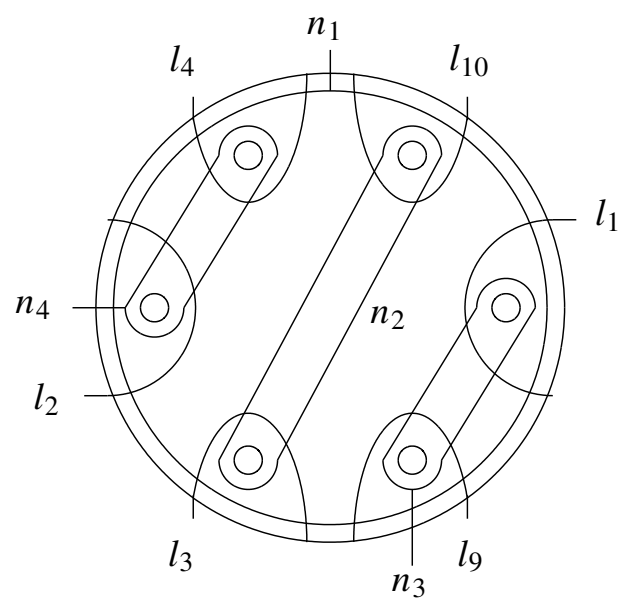

Figure 15 


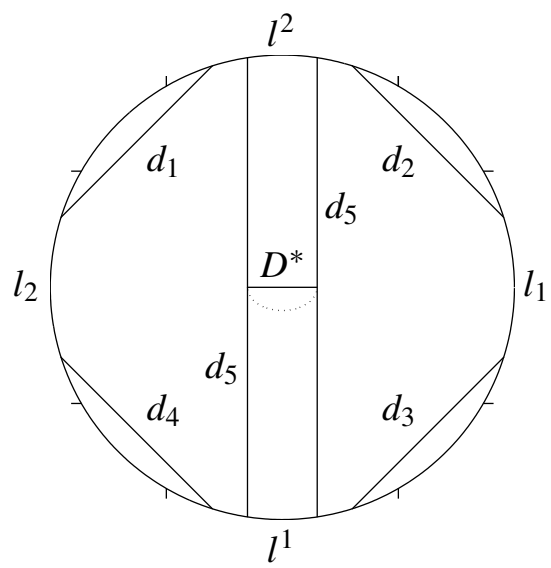

Figure 16

of $\partial J^{2}-\partial A^{*}$. Thus there is a $\partial$-compressing disk $D^{*}$ of $S \cap J^{2}$ as in Figure 16 . By doing a surgery on $F$ along $D^{*}$, we can obtain a surface $F^{\prime}$ isotopic to $F$ such that $\left|F^{\prime} \cap W\right|=|F \cap W|,\left|F^{\prime} \cap F_{2}\right|=\left|F \cap F_{2}\right|$, and $\left|\left(F^{\prime} \cap M_{1}-X\left(F^{\prime}\right)\right) \cap W^{\prime}\right|<$ $\left|\left(F \cap M_{1}-X(F)\right) \cap W^{\prime}\right|$, a contradiction.

Suppose instead that $v\left(d_{1}\right)<v\left(d_{2}\right)$. Set $k=\mid v\left(d_{2}\right)-v\left(d_{1} \mid\right.$ and $n=\operatorname{gcd}(k, k+$ $v\left(d_{5}\right)$. Then $\partial\left(S \cap J^{2}\right)$ contains $v\left(d_{1}\right)$ trivial components and $n$ components $c$ isotopic to $\alpha^{p} \beta^{q}$, where $|q|=\left(k+v\left(d_{5}\right)\right) / n$ and $|p|=k / n$. By construction, $p>0$ if and only if $q>0$. (See Figure 2.) That means that $c$ is not null homotopic in $J^{2}$. By the proof of Lemma 5.3, we can obtain a surface $F^{\prime}$ isotopic to $F$ such that $C\left(F^{\prime}\right)<C(F)$, a contradiction.

Finally, suppose that $v\left(d_{1}\right)>v\left(d_{2}\right)$, and define $k$ as in the previous case. By the preceding argument, $\partial\left(S \cap J^{2}\right)$ contains $v\left(d_{2}\right)$ trivial components and $n$ components $c$ isotopic to $\alpha^{p} \beta^{q}$, where $|q|=\left(k+v\left(d_{5}\right)\right) / n$ and $|p|=k / n$. If $c$ is not nullhomotopic in $J_{2}$, then by the preceding argument, we can obtain a surface $F^{\prime}$ isotopic to $F$ so that $C\left(F^{\prime}\right)<C(F)$, a contradiction. Assume that $q=-2 p$. Then $v\left(d_{5}\right)=v\left(d_{1}\right)-v\left(d_{2}\right)$. Since $v\left(d_{5}\right)=v\left(d_{1}\right)+v\left(d_{2}\right), v\left(d_{2}\right)=0$ and $v\left(d_{5}\right)=v\left(d_{1}\right)$. Thus $F_{1} \cap F$ is as in Figure 15 with $v\left(n_{2}\right)=v\left(n_{3}\right)=v\left(n_{4}\right)$ and $v\left(n_{1}\right)=0$. This completes the analysis when $v\left(f_{3}\right)=v\left(f_{6}\right)=0$.

If $v\left(f_{3}\right)=v\left(f_{6}\right) \neq 0$ in Figure 11, right, there are two subcases:

Suppose first that $v\left(d_{1}\right) \leq v\left(d_{2}\right)$. Then $S \cap F_{1}$ contains $\min \left(v\left(d_{1}\right), v\left(f_{3}\right)\right)$ components isotopic to $\partial E_{1}$. If $v\left(d_{1}\right) \geq v\left(f_{3}\right)$, we can obtain, by the same argument as in the preceding case, a surface $F^{\prime}$ isotopic to $F$ such that $C\left(F^{\prime}\right)<C(F)$, a contradiction. Assume that $v\left(d_{1}\right)<v\left(f_{3}\right)$, then $S \cap F_{1}$ contains $v\left(d_{1}\right)$ components isotopic to $\partial E_{1}$. Now $2 v\left(f_{1}\right)=v\left(d_{1}\right)+v\left(d_{2}\right)$. By assumption, $v\left(f_{1}\right)=$ $v\left(f_{3}\right)+v\left(f_{4}\right)$. Thus $v\left(d_{1}\right)<v\left(d_{2}\right)$. Then, by the proof of Lemma 5.3, $\partial\left(S \cap J^{2}\right)$ contains $\operatorname{gcd}\left(k, k+v\left(d_{5}\right)\right)$ components each of which is isotopic to $\alpha^{p} \beta^{q}$, where 
$|q|=\left(k+v\left(d_{5}\right)\right) / n$ and $|p|=k / n$ (here again we have set $k=\mid v\left(d_{2}\right)-v\left(d_{1} \mid\right.$ and $n=\operatorname{gcd}\left(k, k+v\left(d_{5}\right)\right)$. If $q \neq-2 p$, then by the proof of Lemma 5.3, there is in $H_{K}$ an essential closed surface $F^{\prime}$ isotopic to $F$ such that $C\left(F^{\prime}\right)<C(F)$, a contradiction. Since $y=v\left(d_{2}\right)-v\left(d_{1}\right)=2\left(v\left(f_{1}\right)-v\left(d_{1}\right)\right)>2\left(v\left(f_{1}\right)-v\left(f_{3}\right)\right)=2 v\left(f_{5}\right)$, we conclude that $v\left(d_{5}\right)=2 v\left(f_{5}\right)$. Thus $q \neq-2 p$.

If, on the other hand, $v\left(d_{1}\right)>v\left(d_{2}\right)$, then $S \cap F_{1}$ contains $\min \left(v\left(d_{2}\right), v\left(f_{3}\right)\right)$ components isotopic to $\partial E_{1}$. If $v\left(d_{2}\right) \geq v\left(f_{3}\right)$, then by the same argument as before the pattern of $F \cap F_{1}$ is as in Figure 15, with $v\left(n_{1}\right)=v\left(f_{3}\right)$ and $v\left(n_{2}\right)=$ $v\left(n_{3}\right)=v\left(n_{4}\right)$. But then we see that it is impossible to have $v\left(d_{1}\right)<v\left(f_{3}\right)$.

Lemma 5.5. $H_{K}$ contains no closed essential surface.

Proof. Suppose, to the contrary, that $H_{K}$ contains an essential closed surface $F$ such that the complexity $C(F)$ is minimal among all surfaces isotopic to $F$. By Lemma 5.1, the pattern of $F \cap F_{2}$ is as in one of the diagrams of Figure 8. Furthermore, $v\left(m_{2}\right)=v\left(m_{3}\right)=0$ for any case. By Lemmas 5.3 and 5.4, the pattern of $F \cap F_{1}$ is as in one of Figures 12 and 15. Furthermore, $v\left(n_{2}\right)=v\left(n_{3}\right)=v\left(n_{4}\right)$ for any case. By Lemma 5.0, $v\left(n_{1}\right)+v\left(n_{2}\right)=v\left(m_{1}\right)$.

In $M_{2}$, the pattern of $F \cap F_{1}$ can be labeled as in one of the diagrams on the top row of Figure 17, and the pattern of $F \cap F_{2}$ can be labeled as in Figure 17, bottom.
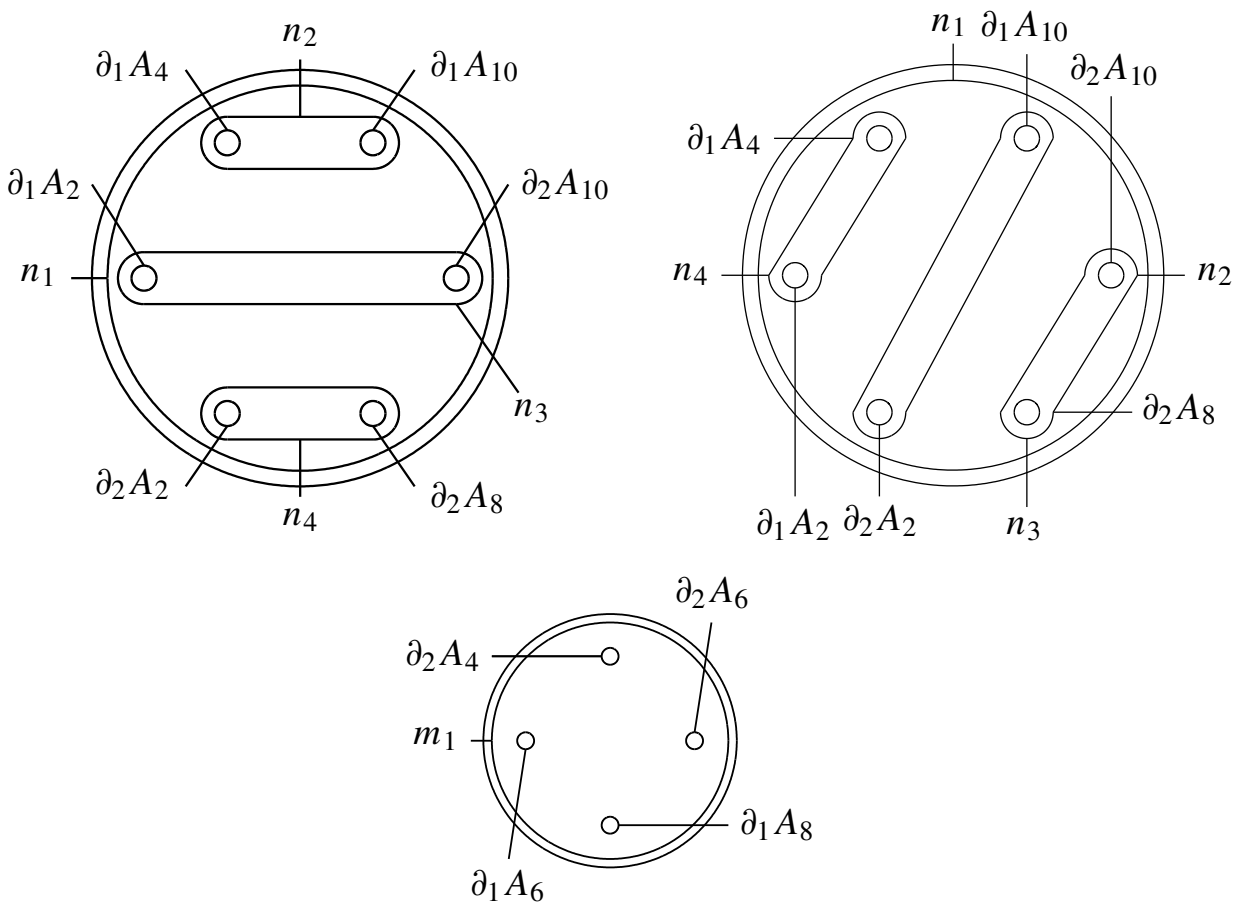

Figure 17 


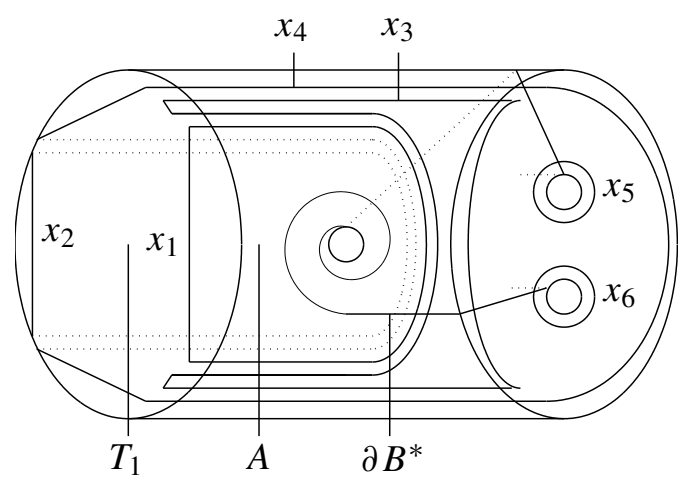

Figure 18

Note that $W_{2}, W_{4}, W_{8}, W_{10}$ separate $M_{2}$ into four solid tori $J^{1}, J^{2}, J^{4}, J^{5}$ and a handlebody of genus two $H^{\prime}$ such that $A_{2 i} \subset J^{i}$ for $i=1,2,4,5$ and $A_{6} \subset H^{\prime}$. Let $S=F \cap H^{\prime}$.

Now we claim that $v\left(n_{2}\right)=v\left(n_{3}\right)=v\left(n_{4}\right)=0$. There are two cases:

Case 1. The pattern of $F \cap F_{1}$ is as in Figure 17, top left. Now each component of $\partial S$ is contained in one of the eight families $x_{1}, \ldots, x_{8}$ as in Figures 18 and 19 , where the boundary components of $\partial S$ contained in $\bigcup_{i=1}^{4} x_{i}$ are produced by cutting along the arcs in $F \cap\left(W_{2} \cup W_{4} \cup W_{8} \cup W_{10}\right)$ whose endpoints lie in $m_{1} \cup n_{1}$ and the components of $\partial S$ contained in $x_{7} \cup x_{8}$ are produced by cutting along the arcs whose endpoints lie in $n_{2} \cup n_{3} \cup n_{4} \cup m_{1}$, and each component in $x_{5} \cup x_{6}$ is isotopic to one component of $\partial A_{6}$. Each component lying in $x_{3} \cup x_{4}$ is trivial in $\partial H^{\prime}$. By observation, there are two disks $D^{1}$ and $D^{2}$ in $\partial H^{\prime}$ such that $\partial D^{i}=b_{i} \cup b_{i}^{\prime}$, where $b_{i} \subset F_{1}$ and $b_{i}^{\prime} \subset S$ as in Figure 19. Back to $M_{2}, D^{1}$ and $D^{2}$ are as in Figure 12. Thus by doing surgeries on $F$ along $D^{1}$ and $D^{2}$, we can obtain a surface $F^{\prime}$ isotopic to $F$ such that $\left|F^{\prime} \cap W\right|=|F \cap W|,\left|F^{\prime} \cap F_{2}\right|=\left|F \cap F_{2}\right|$ and $\left|\left(F^{\prime} \cap M_{1}-X\left(F^{\prime}\right)\right) \cap W^{\prime}\right|<\left|\left(F \cap M_{1}-X(F)\right) \cap W^{\prime}\right|$, contradicting minimality.

Case 2. The pattern of $F \cap F_{1}$ is as in Figure 17, top right. This is similar to Case 1.

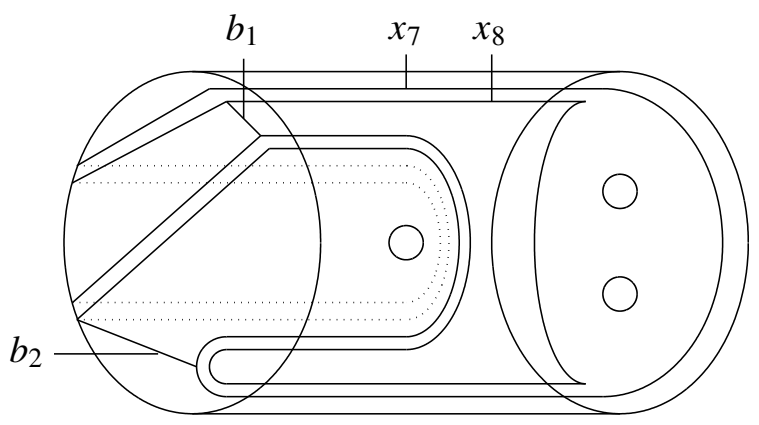

Figure 19 

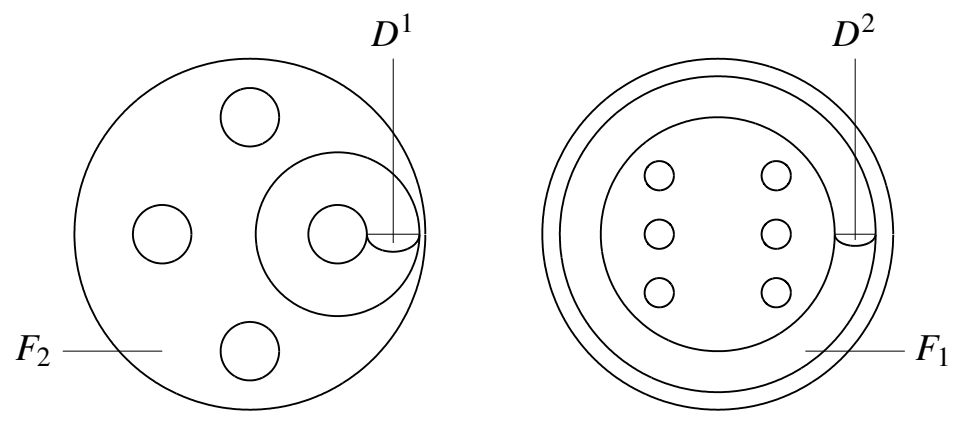

Figure 20

Now $v\left(n_{2}\right)=v\left(n_{3}\right)=v\left(n_{4}\right)=0$ and $\partial S$ is as in Figure 18. By construction, there is a disk $B^{*}=H^{\prime} \cap D_{6 *}$ in $H^{\prime}$ such that $\partial B^{*}$ intersects each component in $x_{1} \cup x_{2} \cup x_{5} \cup x_{6}$ in only one point as in Figure 18 . Thus $S \cap B^{*}$ offers a $\partial$-compressing disk $D^{*}$ of $S$ such that $D^{*}$ is disjoint from $\AA_{6}$. We denote by $A$ the annulus bounded by an outermost component of $x_{1}$, say $e_{1}$, and an outermost component of $x_{2}$, say $e_{2}$, in $\partial H^{\prime}$, and $T_{1}$ the punctured torus bounded by an outermost component of $x_{1}$ and an outermost component of $x_{2}$ in $\partial H^{\prime}$ as in Figure 18. Now if $\partial D^{*} \cap \partial H^{\prime}=$ $a \subset A$, then $e_{1} \cup e_{2}$ bounds an annulus in $S$ parallel to $A$. This means that one component of $F \cap M_{2}$ is parallel to $\partial H \cap M_{2}$.

Let $X_{0}(F)$ be a union of components in $F \cap M_{2}$ parallel to $\partial H \cap M_{2}$ or $A_{6}$, and set $S=\left(F \cap M_{2}-X_{0}(F)\right) \cap H^{\prime}$. Then $\left(F \cap M_{2}-X_{0}(F)\right) \cap H^{\prime} \cap B^{*}$ offers a $\partial$-compressing disk, also denoted by $D^{*}$, of $S$ such that $\partial D^{*} \cap \partial H^{\prime}=a$.

We claim each component of $S$ is isotopic to one component of $\partial A_{6}$. There are five possibilities:

(1) The two endpoints of a lies in $x_{5}\left(x_{6}\right)$. Then $D^{*}$ can be moved to be $D^{1}$ as in Figure 20(a), Thus by doing a surgery on $F$ along $D^{1}$, we can obtain a surface $F^{\prime}$ isotopic to $F$ such that $\left|F^{\prime} \cap W\right|=|F \cap W|,\left|F^{\prime} \cap F_{2}\right|<\left|F \cap F_{2}\right|$, a contradiction.

(2) The two endpoints of a lies in $x_{1}\left(x_{2}\right)$. Then $D^{*}$ can be moved to be $D^{2}$ as in Figure 20(b), contradicting the minimality of $|F \cap W|$.

(3) One endpoint of a lies in $x_{5}$ and the other lies in $x_{6}$. Since $\partial B^{*}$ intersects $\bigcup_{i=1}^{6} x_{i}$ in the order $x_{6}, x_{3}, x_{1}, x_{2}, x_{4}, x_{5}$, there is by the argument in (1) an outermost component of $S \cap B^{*}$ in $B^{*}$, say $b$, which, together with an $\operatorname{arc} b^{*}$ in $\partial H^{\prime}$, bounds an outermost disk $D$ such that $\partial_{1} b$ is contained in $x_{5}, \partial_{2} b$ is contained in $x_{6}$ and $b^{*}$ intersects $A_{6}$ in an arc. Since $S$ is incompressible, by the standard argument, the component of $S$ containing $b$ is parallel to $A_{6}$, a contradiction.

(4) One endpoint of a lies in $x_{1}$ and the other lies in $x_{2}$. Then $\partial_{1} a \subset c_{1}$ and $\partial_{2} a \subset c_{2}$, where $c_{1}$ is a component of $x_{1}$ and $c_{2}$ is a component of $x_{2}$. We denote again by $A$ the annulus bounded by $c_{1}, c_{2}$ in $\partial H^{\prime}$ and by $T_{1}$ the punctured torus bounded by 
$c_{1}, c_{2}$ in $\partial H^{\prime}$. Note that $a$ is disjoint from $\AA_{6}$ and $A_{6} \subset T_{1}$. Hence $a \subset A$. By the preceding argument, the component of $F \cap M_{2}$ consisting of $c_{1}$ and $c_{2}$ is parallel to $\partial H \cap M_{2}$. By the definition of $S$, this is impossible.

(5) One endpoint of a lies in $x_{1} \cup x_{2}$ and the other lies in $x_{5} \cup x_{6}$. Since $S$ is incompressible, each component $c$ of $x_{3} \cup x_{4}$ bounds a disk $D_{c}$ in $S$ parallel to a disk $D_{c}^{*}$ on $\partial H^{\prime}$; see Figure 18. Let $S^{*}=S-\cup_{c \in x_{3} \cup x_{4}} D_{c}$. Note that $\partial B^{*}$ intersects $\bigcup_{i=1}^{6} x_{i}$ in the order $x_{6}, x_{3}, x_{1}, x_{2}, x_{4}, x_{5}$. Hence each component of $S \cap B^{*}$ is an arc $b$ such that $\partial_{1} b \subset x_{1} \cup x_{2}$ and $\partial_{2} b \subset x_{5} \cup x_{6}$. Otherwise there would be an outermost component $b^{*}$ of $S^{*} \cap B^{*}$ in $B^{*}$ such that $\partial b^{*}$ is as in one of the above four cases, a contradiction.

Each component of $S \cap B^{*}$ is an arc $b$ such that $\partial_{1} b \subset x_{1} \cup x_{2}$ and $\partial_{2} \subset x_{5} \cup x_{6}$. Set $H^{*}=H^{\prime}-B^{*} \times(0,1)$ and $S^{* *}=S^{*}-B^{*} \times(0,1)$, where $B^{*} \times I$ is a regular neighborhood of $B^{*}$ in $H^{\prime}$. Then $H^{*}$ is a solid torus. Since each component of $x_{1} \cup x_{2} \cup x_{5} \cup x_{6}$ intersects $\partial B^{*}$ in one point, each component $h$ of $\partial S^{* *}$ is obtained by doing a band sum of one component $h_{1}$ of $x_{5} \cup x_{6}$ and one component $h_{2}$ of $x_{1} \cup x_{2}$ along a component of $S^{*} \cap B^{*}$. Since $h_{1}=1 \in \pi_{1}(H)$, we have $h_{2} \neq 1 \in \pi_{1}(H)$, so $h \neq 1 \in \pi_{1}\left(H^{*}\right)$. Recall the disk $B_{2}$ in $H$ defined in Section 2. The intersection $B_{2} \cap H^{\prime}$ is a planar surface $P$ such that one component of $\partial P$, say $\partial_{1} P$, is disjoint from $A_{6}$, and the other components of $\partial P$ lie in $\AA_{6}$. Furthermore, $\partial_{1} P$ intersects each component in $x_{1} \cup x_{2}$ in one point. Hence $P-B^{*} \times(0,1)$ is a properly embedded disk in $H^{*}$ intersecting each component of $\partial S^{* *}$ in one point. This means that each component of $S^{* *}$ is an annulus $A$ parallel to each component of $\partial H^{*}-\partial A$.

Suppose that $D$ is a $\partial$-compressing disk of $A$ in $H^{*}$ such that the $\operatorname{arc} \alpha=D \cap \partial H^{*}$ lies on the annulus $A^{*}$ on $\partial H^{*}$ which contains the disk $A_{6}-B^{*} \times(0,1)$. Then $D$ is disjoint from $x_{3} \cup x_{4}$. Since the disk $D^{*}=B^{*} \times\{0,1\} \cup\left(A_{6}-B^{*} \times(0,1)\right)$ intersects $\partial A^{*}$ in two arcs, $D$ can be moved to have the arc $\alpha$ lying on $A^{*}-D^{*}$. Furthermore, since each component $h$ of $\partial S^{* *}$ is obtained by doing a band sum of one component $h_{1}$ of $x_{5} \cup x_{6}$ and one component $h_{2}$ of $x_{1} \cup x_{2}$, we may assume that $\partial \alpha \subset x_{1} \cup x_{2}$. Hence $D$ is also a $\partial$-compressing disk of $S^{*}$ in $H^{\prime}$. By the preceding argument, this is impossible.

Also by the preceding argument, if one component of $F \cap\left(F_{1} \cup F_{2}\right)$ is parallel to $\partial E_{1}$ or $\partial E_{2}$ then it is parallel to $\partial H$. Suppose that each component of $F \cap\left(F_{1} \cup F_{2}\right)$ is isotopic to one component of $\partial A_{i}$. By the minimality of $C(F), F$ is disjoint from $W_{i}$ for $i \neq 6$ and $F$ is also disjoint from $\overline{\partial N\left(B^{*} \cup A_{6}\right)-\partial H^{\prime}}$ in $H^{\prime}$. Thus each component of $F \cap M_{j}$ is an annulus parallel to $A_{i}$ for some $i$. That means that $F$ is isotopic to $T$, a contradiction.

Proof of Proposition 3.0. The proposition follows immediately from Lemmas 4.1, 4.3, 4.4 and 5.5 and [Scharlemann and $\mathrm{Wu}$ 1993, Theorem 1]. 


\section{Acknowledgment}

We thank Professor Fengchun Lei for helpful discussions, and the referee for a careful reading of the paper and for pointing out a mistake in an earlier version.

\section{References}

[Culler et al. 1987] M. Culler, C. M. Gordon, J. Luecke, and P. B. Shalen, "Dehn surgery on knots", Ann. of Math. (2) 125:2 (1987), 237-300. MR 88a:57026 Zbl 0633.57006

[Gordon 1997] C. M. Gordon, "Combinatorial methods in Dehn surgery”, pp. 263-290 in Lectures at KNOTS '96 (Tokyo 1996), Ser. Knots Everything 15, World Sci. Publ., River Edge, NJ, 1997. MR 98m:57020 Zbl 0940.57022

[Hatcher 1982] A. E. Hatcher, "On the boundary curves of incompressible surfaces", Pacific J. Math. 99:2 (1982), 373-377. MR 83h:57016 Zbl 0502.57005

[Jaco 1984] W. Jaco, "Adding a 2-handle to a 3-manifold: an application to property R", Proc. Amer. Math. Soc. 92:2 (1984), 288-292. MR 86b:57006 Zbl 0564.57009

[Lackenby 2002] M. Lackenby, "Attaching handlebodies to 3-manifolds”, Geom. Topol. 6 (2002), 889-904. MR 2003h:57027 Zbl 1021.57010

[Qiu 2000] R. Qiu, "Incompressible surfaces in handlebodies and closed 3-manifolds of Heegaard genus 2”, Proc. Amer. Math. Soc. 128:10 (2000), 3091-3097. MR 2000m:57026 Zbl 0965.57014

[Qiu and Wang 2004] R. Qiu and S. Wang, "Simple, small knots in handlebodies", Topology Appl. 144:1-3 (2004), 211-227. MR 2006a:57008 Zbl 1055.57009

[Qiu and Wang 2005] R. Qiu and S. Wang, "Small knots and large handle additions", Comm. Anal. Geom. 13:5 (2005), 939-961. MR 2216147

[Scharlemann and Wu 1993] M. Scharlemann and Y. Q. Wu, "Hyperbolic manifolds and degenerating handle additions", J. Austral. Math. Soc. Ser. A 55:1 (1993), 72-89. MR 94e:57019 Zbl 0802. 57005

[Thurston 1982] W. P. Thurston, "Three-dimensional manifolds, Kleinian groups and hyperbolic geometry”, Bull. Amer. Math. Soc. (N.S.) 6:3 (1982), 357-381. MR 83h:57019 Zbl 0496.57005

Received June 13, 2005. Revised January 14, 2006.

\section{RUIFENG QIU}

DePaRTMENT OF MATHEMATICS

DALIAN UNIVERSITY OF TECHNOLOGY

DALIAN 116022

CHINA

qiurf@dlut.edu.cn

\section{SHICHENG WANG}

Department of Mathematics

PEKING UnIVERSITY

BEIJING 100871

CHINA

wangsc@math.pku.edu.cn 